\title{
Body illusions for mental health: a systematic review
}

Marta Matamala-Gomez ${ }^{1}$, Antonella Maselli ${ }^{2}$, Clelia Malighetti ${ }^{3}$, Olivia Realdon ${ }^{1}$, Fabrizia Mantovani $^{1}$, Giuseppe Riva ${ }^{3,4}$

${ }^{1}$ University of Milano-Bicocca, "Riccardo Massa" Department of Human Sciences for Education, Milan, Italy.

${ }^{2}$ Institute of Cognitive Sciences and Technologies (ISTC), National Research Council (CNR), Rome, Italy.

${ }^{3}$ Department of Psychology, Catholic University of Milan, Milan, Italy.

${ }^{4}$ Applied Technology for Neuro-Psychology Laboratory, Istituto Auxologico Italiano, IRCCS, Milan, Italy.

\section{Corresponding author:}

Marta Matamala-Gomez

marta.matamala10@gmail.com

\section{Abstract}

Body illusions (BIs) refer to altered perceptual states where the perception of the self-body significantly deviates from the configuration of the physical body, for example, in aspects like perceived size, shape, posture, location, and sense of ownership. Different established experimental paradigms allow to temporarily induce such altered perceptual states in a predictable and systematic manner. Even though there is evidence demonstrating the use of BIs in clinical neuroscience, to our knowledge, this is the first systematic review evaluating the effectiveness of BIs in healthy and clinical populations. This systematic review examined the use of BIs in the healthy and clinical populations, and review how BIs can be adopted to enhance mental health in different mental illness conditions. The systematic review was conducted following the PRISMA guidelines. Of the 8086 studies identified, 189 studies were included for full-text analyses. Seventy-seven studies used BIs in clinical populations. Most of the studies using BIs with clinical populations used body illusions toward a body part, modulating the external aspects of body representation. Even though clinical studies showed the positive effects of BIs to improve mental illness conditions, future technologies using BIs targeting both the external (exteroceptive) and the internal (interoceptive) aspects of body representations can further improve the efficacy of this approach.

Keywords: body illusions, mental health, mental illness, body representation, body matrix.

\section{Introduction}

The World Health Organization has defined mental health as "a state of well-being in which every individual realizes his or her own potential, can cope with the normal stresses of life, can work 
productively and fruitfully, and is able to make a contribution to her or his community." Gilmour (2014: p.1). In relation to this, mental health has been related not only to the absence of mental illness, but also by three core components: (1) emotional well-being, referring to feelings of happiness and satisfaction with life; (2) psychological well-being, referring to the positive physical functioning that enhance self-realization; and (3) social well-being, positive societal functioning and positive relationships (Westerhof \& Keyes, 2010). Then, mental health is not referring only to psychiatric disorders such as anxiety and depression, but to a wide range of mental disorders that influence peoples' well-being and social interactions such as chronic pain, neurological disorders that may lead to motor o perceptual impairments, psychological disorders that may alter behaviour and social interactions, or physical disorders associated with conditions like in patients with eating disorders or met in amputees.

It is known that an accurate perception of one-self and the surrounding environment are both key elements to reach mental health and well-being (Taylor \& Brown, 1988). After suffering a mental disorder, both can be distorted. Alterations in body representations had been found in patients with a number of neurological disorders: eating disorders, chronic pain (Tsay et al., 2015), psychiatric pathologies such as schizophrenia (Katharine N. Thakkar et al., 2011), recurrent outof-body experiences (OBE) (Blanke \& Mohr, 2005), phantom limb sensations in amputees (Giummarra et al., 2007), and disturbed sensations of limb ownership like somatoparaphrenia and anosognosia (Berlucchi and Aglioti 1997; Vallar and Ronchi 2009).

Neuropathological studies of conditions involving abnormal body perception have indeed provided the first insights on the flexible and dynamic nature of self-body perception emerging from the synergistic activity of widespread brain networks. Based on the numerous report phantoms limbs sensations and phantom pain in amputees, Melzack (1990) suggested that phantom sensations should arise from the "same brain processes that underlie the experience of the body when it is intact", which occur in a neural network encompassing several brain areas (the "neuromatrix"). Melzack also advanced the idea "the feeling that all the parts of the body are uniquely one's own" - i.e. the sense of body ownership, which is impaired in somatoparaphrenic patients - is generated by activity in the neuromatrix. This vision was later supported by the evidence that disturbed sensations of limb ownership are typically associated with brain damages in multisensory integration areas (Bisiach et al., 1991; Vallar \& Ronchi, 2009). Similar is the case of OBE of neurological origin, where altered perceptions of self-location (in which the spatial unity between the physical body and the perceived self is broken) have been associated with abnormal activity in the temporal parietal junction (TPJ) and with the resulting failure to integrate somatosensory, visual and vestibular information into a coherent body representation (Blanke, 2012; Blanke and Mohr, 2005). 
Interestingly, altered body perceptions analogous to those reported in pathological conditions could be induced in healthy individuals using controlled experimental paradigms. All experimental manipulations used to induce body illusions (BIs) consist in exposing participants to multisensory conflicts (see Section 2 for a detailed review). For example, in kinesthetic illusions illusory sensation of extending on bending a joint are induced by applying tendon vibrations that evoke muscle spindle activations similar to those generated during movement execution (Goodwin et al., 1972). In the rubber hand illusion (RHI) (M. Botvinick \& Cohen, 1998; Kammers et al., 2009), participants can temporary misattribute body ownership to an external object (the rubber hand) as a consequence of seeing the rubber hand being touched (or moving) exactly as they feel the real hand being touched (or moving doing). By combining and adapting similar manipulations, it is possible to systematically induce sensations of body deformations, like elongating noses (Lackner \& Levine, 1978) and supernumerary arms (Guterstam et al., 2011), or to distort the perceived location of limbs (Maselli \& Slater, 2014; Zopf et al., 2011), the perceived body posture (Bergström et al., 2016), as well as self-location like in experimentally induced OBE (Ehrsson, 2007; Lenggenhager, 2007).

Not only, experimental BIs have brought tremendous insights into the nature of self-perception, showing how self-body perception is a dynamic and extremely flexible emerging percept, but demonstrated how altered body-perception states can have important impact on perception, behaviour and cognition, particularly for the case of body ownership illusions (BOIs). While undergoing a BOI towards an artificial object (e.g., a rubber or a virtual hand), the physiological state of the body (e.g. the body temperature) (Moseley, Olthof, et al., 2008; Tieri et al., 2017) and the neurophysiological response to external stimuli (e.g. tactile, thermal and noxious stimuli) (Barnsley et al., 2011; Folegatti et al., 2009; Llobera \& Slater, 2013) are temporarily altered, and the temporal constraints for the integration of independent bodily stimuli get looser (Maselli et al., 2016). Ownership illusions have been shown to have also an important impact on motor control (Gonzalez-Franco et al., 2020; Kilteni et al., 2013) and cognitive aspects of behaviour (Maister et al., 2015).

There is large consensus on the fact that BIs are the results of multisensory integration processes (Blanke, 2012; Graziano \& Botvinick, 2002), in which the brain infers in a Bayesian fashion the most probable configuration of the body based on the incoming sensory information streamed by the different sensory channels. For BIs to arise and be sustained, however it is crucial that the sensory conflict is not too large and can be "solved" within the limits of the overall knowledge of human anatomy and the spatiotemporal constraints governing physical entities (Kilteni et al., 2015; Makin et al., 2008). Vast experimental evidence, have also shown that while self-body perception is extremely flexible and malleable, it need to satisfy minimal anatomical constraints in terms of shape and body configurations (Lloyd, 2007; Tsakiris, 2010). 
Theoretical frameworks based on Bayesian inference approaches have been proposed that describe ownership illusions as the results of an inference process in which the brain attributed visual information from the fake body (which should comply with previous semantic knowledge about the human body) and somatosensory stimuli from the physical body to the same one entity: one's own body (Kilteni et al., 2015; Samad et al., 2015). Complementary, the impact of ownership illusions on perception and behaviour has been explained as resulting from the minimization of prediction errors, within the general framework of predictive coding and the free energy principle (Brown \& Friston, 2012; Rao \& Ballard, 1999). For example, the conflict arising from seeing the fake body having different appearance and spatial features with respect to what experienced through touch and proprioception, could be "explained way" (i.e. minimizing) by updating the inferred state of the real hand, e.g. its perceived location drifts towards the fake hand (Samad \& Shams, 2018), or by suppressing the processing of somatosensory sensations (Zeller et al., 2014).

More in general, Moseley and colleagues have introduced the idea a 'body matrix, described as a dynamic and flexible supramodal representation of the body and its surrounding space, emerging from a widespread neural activity encompassing an extended neural network of brain areas. (Gallace and Spence., 2014; Moseley, Gallace, \& Spence, 2012; Sedda, Tonin, Salvato, Gandola, $\&$ Bottini, 2016). More specifically, the body matrix is considered as a high-level representation of the self in the environment which serves the function of integrating and interpreting bodily signals and sensory stimuli from the environment into a coherent framework. In this respect, the body-matrix define a self-body model that allows the top-down predictive processes required to infer the causes of incoming (bottom-up) sensory signals and to drive behaviour during the interaction with the environment (Friston, Daunizeau, Kilner, \& Kiebel, 2010) (Barrett, 2017; Riva et al., 2019). The 'body matrix' in essence is conceived as a prerequisite that allows for efficient self-regulation and adaption to the dynamic environment, and as such it allows for adaptation to profound changes in the anatomical, postural and conceptual representations of our own bodies (Moseley et al., 2012).

Based on the large body of evidence from BI experiments, the "body matrix" view holds that it is possible to modulate different components of the body matrix through ad-hoc controlled experimental manipulations. As an extension, it entails the idea that through the use of BIs it is possible to intervene on the body matrix, when the latter has been distorted due to suffering a mental disorder or mental illness, to recover its functional structure (add Moseley et al. 2012, Riva et... 2019). Here we provide a systematic review of the use of BIs in the healthy and clinical populations, and review how BIs can be adopted to enhance mental health in different mental illness conditions: motor disorders, pain disorders, psychological and psychiatric disorders, amputation, and other neurological disorders. 


\section{Body illusions}

Body illusions (BIs) refer to altered perceptual states where the perception of the self-body significantly deviates from the configuration of the physical body, for example in aspects like perceived size, shape, posture, location and sense of ownership. Different established experimental paradigms allow to temporarily induce such altered perceptual states in a predictable and systematic manner. These paradigms are based on ad-hoc manipulations of bodily stimuli so that, by delivering conflicting information about the body, illusory experiences arise as the brain "looks for" an explanatory solution to the conflict (Hohwy \& Paton, 2010). For example, to explain away the fact that one keeps touching the tip of her nose, while felling that her elbow is extending, the illusory sensation of an elongating nose arises (Lackner, 1988). Analogously, in the rubber hand illusion (RHI) (Botvinick \& Cohen, 1998), illusory body ownership towards the rubber hand, emerges to "explain away" the highly unlikely event that tactile sensations felt on one's hidden hand are perfectly congruent with the touch seen on a rubber hand placed in a position congruent with the hidden hand.

The fact that BIs can be triggered in healthy individual through relatively simple experimental manipulations (e.g. visuo-tactile or visuo-motor correlations), supports the overall view that selfbody perception is built dynamically on the base of multisensory integration processes and of the prior knowledge we have about the human body. Hence, even though we believe that the own internal body representation is stable, it is in truth highly malleable. Through BIs subjects can embody fake body parts or whole fake bodies, which are therefore processed as belonging to or substituting their physical body. The sense of embodiment has been described as the sense of the own body (Arzy et al. 2006), embedding three components: (1) body ownership, (2) self-location, and the (3) sense of agency (Longo et al. 2008; Riva 2018). More specifically, body ownership refers to the sense of owning a body, self-location refers to the perceived location of the self in space, and the sense of agency is related to the sense of controlling the motor actions, and motor intentions of the physical body (Longo et al. 2008; Riva 2018). A vast body of experimental studies demonstrated that it is possible to selectively manipulate these components through appropriate multisensory manipulations, as well as it is possible to modify the perceived physical configuration of the self-body. In the following, we present an overview of different types of body illusions used in the field of clinical and psychological neuroscience.

\section{Body illusions for body representation}

\section{Kinesthetic illusions and body distortions illusions}


An illustrative example of body illusions are kinesthetic illusions, in which illusory perception of self-motion occurs while being in a static position (Goodwin et al., 1972). Subjects therefore perceived unveridical postures, which in some cases can even violate anatomical constraints of the physical body. These illusions are typically induced in blindfolded subjects, by applying a mechanical vibration to a tendon muscle of a physically immobilized joint, in specific frequency ranges that can trigger activations of the muscle spindles similar to the activity produced during real movement

Illusory movements, when coupled with self-touch, can lead to a modulation in the implicit body representation creating body distortion illusions of the body parts in contact. In this way illusory perceptions of elongating noses (Lackner, 1988) and fingers (de Vignemont et al., 2005), or shrinking waists (Ehrsson, Kito, et al., 2005) can be elicited.. A similar illusion of body distortion is the Pinocchio illusion or the phantom nose illusion (Ramachandran \& Hirstein, 1998), in which subjects experienced to have a very long nose through temporal correlations between touching the nose of a person in front of them and the touch delivered from the experimenter on their own nose.

\section{Out-of-body-experiences}

Another important type of BIs are the experimentally induced out of body experiences (OBEs). OBEs are described as "an experience in which a person seems to perceive the world from a location outside their physical body" (SJ Blackmore 2017, p.26). OBE are therefore associated with altered states of perceived self-location, in which people perceive their selves outside the physical limits of their body (Blanke et al., 2008). Two major experimental paradigms have been proposed to induce similar transitory perceptual experiences in healthy subjects (Ehrsson, 2007; Lenggenhager et al., 2007). Both are based in the manipulation of the visual perspective: participants wear a head-mounted display (HMD), which streams in real time videos from two cameras registering the back of the participants from a distance (about $2 \mathrm{~m}$ away); then, participants observe their own body from the back, from a third-person perspective (3PP), in contrast with the first-person perspective (1PP), that is the usual perspective of the body when one looks down to their feet. Hence, stimulation illusory OBE of different type can be induced by applying visuo-tactile stimulation in different modalities. In one case, the VT stimulation is applied on theparticipants' back, so that they feel the touch on the back while seeing the synchronous tactile stimulation from a 3PP on the distant body (Lenggenhager, 2007). This induce an an illusory drift in the perceived self-location toward the body seen in the displayed video through the HMD and an associated sense of self-identification with the seen body (Aspell et al., 2009; Blanke et al., 2008; Lenggenhager et al., 2007). The second approach consists in tapping the participants' chest on their chest with a stick, while tapping with another stick the air between 
two cameras; so, the touch is felt on the chest and seen in the corresponding space below the "disembodied" visual perspective, while the self-body is seen from a distance. This results in an illusory experience in which participants feel disownership towards their own body and perceived their selves at the location of their visual perspective (Ehrsson, 2007; Guterstam \& Ehrsson, 2012). In summary, although both types of illusory OBE modulate self-location, they do it in different ways (Maselli, 2015): while the back-stroking paradigm mainly affects the egocentric srepresentatio of self-location (i.e., the egocentric mapping of somatosensory sensation in the external space), the chest stroking paradigm has an impact on the allocentric representation of self-location (i.e., where is the self located in the external space).

Body ownership illusionsThe main aspect to differentiate our own body from other's bodies or objects in the environment lies in the type of sensory input arriving at the body and the later processing in the brain. The processing of somatosensory information (e.g., thermal, tactile, somatosensation, nocioception) is unique to our own body, while it is not available to our brain when it comes to the perception of others' bodies (Longo, 2015). Experimental paradigms for eliciting body ownership illusions all rely on establishing a "connection" between the real body (part) and a fake bodily shaped object via multisensory stimulation.

\section{Rubber Hand Illusion}

The first formal study investigating BOI was conducted by Botvinick and Cohen (1998) who presented the Rubber Hand Illusion (RHI) paradigm for the first time. Participants were presented with a rubber hand placed close by the occluded real hand, and the two hands were stroked synchronously, eliciting the illusory experience that the felt touch was originating from the rubber hand, with an associated recalibration of the perceived position of the real hand towards the rubber hand - known as the proprioceptive drift. This study was soon followed by numerous other experimental works that adopted and modified the RHI paradigm to investigate the perceptual, cognitive and neurophysiological underpinning of the sense of body ownership. It was shown that ownership illusion toward a fake hand (rubber or virtual) could be elicited with other form of multisensory stimulation, like visuomotor correlations where one sees the fake hand moving as the real hand is moving (Kalckert \& Ehrsson, 2012; Sanchez-Vives et al., 2010; Spychala et al., 2020), or even by simple visuo-proprioceptive correlations where a static fake hand is seen colocated with or very close the real hand (Giummarra, Georgiou-Karistianis, et al., 2010; Longo et al., 2008). Other studies have however demonstrated that the RHI can occur only if the fake object to be embodied complies in its visual appearance with the basic anatomical constraints of 
a hand shape (Tsakiris et al., 2010)and also if it is presented in an anatomical plausible configuration (Ehrsson, Holmes, et al., 2005; Lloyd, 2007).

According to a general consensus, the RHI arises as the brain's perceptual system resolves a sensory conflict by integrating independent but synchronous multisensory bodoly related inputs; however, this can occur only under the filter of a top-down cognitive mechanism that inhibits the integration when the external object is anatomically implausible (Matthew Botvinick, 2004; Makin et al., 2008; Tsakiris, 2010).

\section{Mirror Box Illusion}

Another well-known BOI toward a body part used in the clinical population suffering from phantom limb pain or motor disorders, such as arm paresis, is the mirror box illusion (MBI) introduced by Ramachandran and Rodgers-Ramachandran (1996) with the mirror visual feedback (MVF) therapy. In the MVF therapy, a mirror is placed along the sagittal plane of the patient, who places his/her affected limb behind the mirror and the healthy limb on the reflective side of it. In this way, when looking into the mirror in the direction of the affected limb patients could see the reflection of their healthy limb superimposed on the felt location of the affected limb, thereby creating the illusion that the affected limb has been recovered. Hence, while looking at the mirror, if the patient sends motor commands to both hands to perform symmetrical movements, the patient acquires the visual impression that his affected hand responds to his motor command. Later, MVF therapy was mainly used to treat two neurological disorders: chronic pain of central origin, and motor disorders, such as hemiparesis after stroke injury (Ramachandran et al., 2009).

\section{Enfacement Illusion}

The enfacement illusion (EI) has been defined as the subjective illusion of looking at oneself in the mirror when looking at another's person face (Porciello et al., 2018). . The EI can be elicited by showing a picture of another person face in a desktop and providing interpersonal multisensory stimulations (e.g., touching the participant's face and showing a synchronous pattern of stroking of the face in the video) (Sforza et al., 2010). Authors demonstrated that in this way it is possible to manipulate self-face representation, which has been considered a stable construct of the body image difficult to change, by using simple experimental manipulations (Porciello et al., 2018).

\section{Full Body Ownership Illusions}

The sense of ownership illusions has also been induced toward full fake bodies, by using HMDs through which is possible to observe the entire fake body from a first-person perspective and place at the same spatial location as the real body is (Maselli \& Slater, 2013; Petkova \& Ehrsson, 2008; 
S. Serino et al., 2019; Slater et al., 2009). As for the RHI, full body ownership illusions (FBOI) can be achieved through visuo-tactile stimulation (Normand et al., 2011; Petkova \& Ehrsson, 2008; S. Serino et al., 2018), visuo-motor correlations (Serino, Polli, and Riva 2019; Seinfeld et al. 2018), as well as through static visuo-proprioceptive sensory signal (Maselli \& Slater, 2013). Independent of the methodology, recent studies based on the FBOIs demonstrated that the brain can flexibly and dynamically adapt to bodies with different appearance, shapes and posture with important perceptual and cognitive consequences (Maister et al., 2015; Riva \& Dakanalis, 2018).

Altogether, by means of BOIs it is possible to induce the sense of embodiment either toward a fake body part or to a full fake body. As general account, body ownership illusions have been proposed to arise as the result of an inference process in which the brains attribute all the incoming sensory information about the body (visual information from the fake body and somatosensory sensations from the real body) to the same common cause, which is the own body (Kilteni et al., 2015; Samad et al., 2015). Importantly, such inference results can occur only if the incoming sensory information satisfies to some degree prior knowledge about the human body anatomy and about the spatiotemporal correlation across sensory channels.

\section{Neural networks related to body illusions}

It is known that the sense of embodiment is provided by the integration of the sensory signals arriving from our body through the different sensory channels, which is processed a widespread network of brain areas including temporoparietal, motor and premotor, posterior parietal, and extrastriate cortices (Olaf Blanke 2012). However, it is also know that subcomponents of embodiment - like ownership, self-location, and agency - are characterized by different neurophysiological correlates (see Serino et al. 2013).

The neural correlates of these three components of embodiment have been investigated by performing experimental studies using the above described illusory OBEs and BOIs toward fake bodies and body parts (e.g., RHI, MBI, enfacement illusion). Regarding BOIs toward fake body parts, several functional magnetic resonance imaging (fMRI) and positron emission tomography (PET) studies, showed the activation of the bilateral premotor cortex (PMC), the intraparietal sulcus (IPS), the insula, the sensorimotor cortex, and the cerebellum, are linked with ownership illusions towards a body part (Ehrsson, Spence, and Passingham 2004; H. H. Ehrsson, Holmes, and Passingham 2005; Kammers et al. 2009; Kanayama, Sato, and Ohira 2007, 2009; M. Tsakiris et al. 2007). Overall, neuroimaging studies conducted in humans emphasize two main brain region involved in arm ownership illusions, the PMC and the PPC, within an extensive neural network 
involving the insula, the primary somatosensory cortex, the lateral occipital complex, TPJ, the supplementary motor area, the anterior cingulate cortex, and the cerebellum (see Olaf Blanke, Slater, and Serino 2015). Nevertheless, only the activation of the PMC, but no in the IPS, was correlated with the strength of ownership illusion (questionnaire assessment) towards the rubber hand (Ehrsson et al., 2004). Similar results have been found for the case of illusory ownership towards a whole fake body (Gentile et al. 2015; Petkova et al. 2011).

Different brain areas have instead been associated with the changes in self-location occurring during OBEs illusions. However, depending on the type of experimental paradigm adopted (backstroking vs chest stroking) different brain activation have been reported (Maselli, 2015). Ionta et al. (2011) observed that changes in self-locations induced with the back stroking paradigms were associated with the activation of the TPJ and the middle-inferior temporal cortex, but not with the PMC and IPS (Ionta et al. 2011). In this case, functional connectivity analysis have further revealed an enhanced connectivity of the TPJ with a right hemisphere network including the insula and the supplementary motor area (Ionta et al., 2014) Different areas have been instead associated with the changes in self-location induced with the chest stroking paradigms: in this case illusory states correlated with activations in in the hippocampus, posterior cingulate, and IPS areas have been found (Guterstam et al. 2015). These differences are consistent with the view that self-location can be regarded as the blending of two parallel representations, an egocentric mapping of somatosensory sensations into the external space, which corresponds to an extension of the peri-personal space, and an allocentric representation of the location occupied by the self in the environment (Maselli, 2015).

Regarding the sense of agency, researchers have shown that feeling responsible for performing an action activates the anterior insula (Farrer \& Frith, 2002). Moreover, other studies have found that there is a relationship between the sense of agency and the connectivity with prefrontal networks to differentiate themselves from others' actions in a resting-state functional magnetic resonance imaging study (de Bézenac et al., 2018). However, there is evidence demonstrating the role of the supplementary motor area (SMA) contribution in the subjective experience of temporal flow related to goal-directed voluntary actions (Kühn et al., 2013). In addition, it has been shown that either execution and perception of own actions, as well as the perception of others' actions, are functionally connected and processed in common neural networks within the premotor and parietal brain areas (Jackson \& Decety, 2004). Particularly, the inferior parietal cortex has been described as a key brain area in the sense of agency (Jackson \& Decety, 2004). 


\section{Methods}

\section{Data sources and search strategy}

Bibliographical data was collected on Apr 18, 2020, by using the following bibliographic databases: PubMed, EMBASE, Scopus, Web of Science, and PsycINFO. For each database, we used the following combination of research keywords: 1) "mental health" OR "mental disorder" OR "mental illness" OR "mental disease" AND "body illusions"; 2) "mental health" OR "mental disorder" OR "mental illness" OR "mental disease" AND "rubber hand illusion"; 3) "mental health" OR "mental disorder" OR "mental illness" OR "mental disease" AND "mirror therapy"; 4) "mental health" OR "mental disorder" OR "mental illness" OR "mental disease" AND "out of body experiences"; 4) "mental health" OR "mental disorder" OR "mental illness" OR "mental disease" AND "virtual reality"; 5) "mental health" OR "mental disorder" OR "mental illness" OR "mental disease" AND "embodiment", 6) "mental health" OR "mental disorder" OR "mental illness" OR "mental disease" AND "body ownership illusion"; 7) "mental health" OR "mental disorder" OR "mental illness" OR "mental disease" AND "body-swapping". See the detailed search strategy in Table 1. Only full-text available articles were included in our research (conference paper and review articles were excluded), studies citation were retrieved independently for each string of keywords across all databases. The first list of the collected studies during the bibliographic research was exported to Mendeley to remove duplicated studies and then imported to Rayyan (Elmagarmid et al., 2014) for the title and abstract screening, indicating inclusion or exclusion criteria for study selection. The final list of the selected studies for being reviewed was sent to leading experts in the field for suggestion and identification of any missing studies, and no studies were added.

Table 1. Data search strategy

\begin{tabular}{|c|c|c|c|c|c|c|}
\hline \multirow{2}{*}{$\begin{array}{l}\text { Key word/Database } \\
\text { date:13/05/2019 to } 24 / 05 / 2019\end{array}$} & \multicolumn{6}{|c|}{ "mental health" OR "mental disorder" OR "mental illness" OR "mental disease" } \\
\hline & PubMed & Web of Science & PsycINFO & Scopus & Embase & TOTAL \\
\hline Body Illusions & 5 & 1 & 2 & 6 & 32 & 46 \\
\hline Rubber Hand Illusion & 22 & 2 & 22 & 26 & 324 & 396 \\
\hline Mirror therapy & 13 & 3 & 14 & 6 & 294 & 330 \\
\hline Out of Body experiences & 5 & 3 & 52 & 40 & 179 & 279 \\
\hline Virtual reality & 274 & 282 & 893 & 702 & 3268 & 5419 \\
\hline Embodiment & 82 & 126 & 426 & 181 & 630 & 1445 \\
\hline Body swapping & 0 & 4 & 0 & 64 & 4 & 72 \\
\hline Body ownership illusion & 1 & 2 & 1 & 0 & 95 & 99 \\
\hline TOTAL studies in each database & 402 & 423 & 1410 & 1025 & 4826 & \\
\hline TOTAL & & & & & & 8086 \\
\hline Duplicates & & & & & & 1407 \\
\hline NewTOTAL & & & & & & 6679 \\
\hline
\end{tabular}




\section{Eligibility criteria}

The present review aims to discuss different research studies using body illusions for changing body representation in both healthy and clinical populations, presenting some mental illness conditions. Specifically, this systematic review seeks to investigate the influence of BIs in the understanding of mental illness conditions, and how findings from healthy subjects can be moved to clinical populations with mental illness. Bibliographical research was limited to studies using humans and written in English. Further, selected studies had to accomplish the following criteria:

1) Body illusions interventions must have been directed to investigate mental health or mental illness conditions (e.g., pain perception, body representation, psychological or psychiatric conditions, and physiological response) in both healthy and clinical populations. Interventions directed to investigate methodological aspects of how to induce body illusions or to investigate technical aspects of body illusions were excluded.

2) Body illusions interventions must have been directed to a group of healthy or clinical populations, between and within-group study design had been included. Single case or pilot studies with a very small sample size were excluded.

\section{Study selection and data collection}

Two reviewers (M.M.G and C.M) independently analyzed the full text of the final selected articles that accomplished the inclusion criteria. All the reviewers followed the inclusion criteria for study selection. The selected studies were discussed among the two reviewers and discrepancies were solved by consensus or by consulting a third reviewer (A.M). Furthermore, the reviewers contacted all the authors of the studies included in the final selection to identify any missing studies.

\section{Data extraction}

Data extraction was performed by the same two independent reviewers (M.M.G and C.M). Each selected study was coded according to the following thematic categories: (1) name of the authors and age of publication, (2) type of participants; (3) mental health or illness condition; (4) sample size; (5) type of body illusion; (6) type of control condition; (7) type of multisensory correlation (VST or VMC); (8) type of BI assessment; (9) related mental health or mental illness responses (Table 3). The two reviewers followed the coding studies criteria to analyze the final selected studies.

\section{Results}

In the studies research process 8086 studies were found with the above-commented key-words. After removing duplicate studies, a total of 6679 studies were included for the title and abstract 
screening into the Rayyan software. Among the 6979 studies, 6580 studies were excluded because did not accomplish the above described study eligibility criteria. Subsequently, 360 studies were selected for full-text analyses. Of the 360 full text analyzed studies, 189 studies were identified as suitable with the above-described inclusion criteria and were selected for further analyses. See figure 1 for a flow diagram depicting the study selection process.

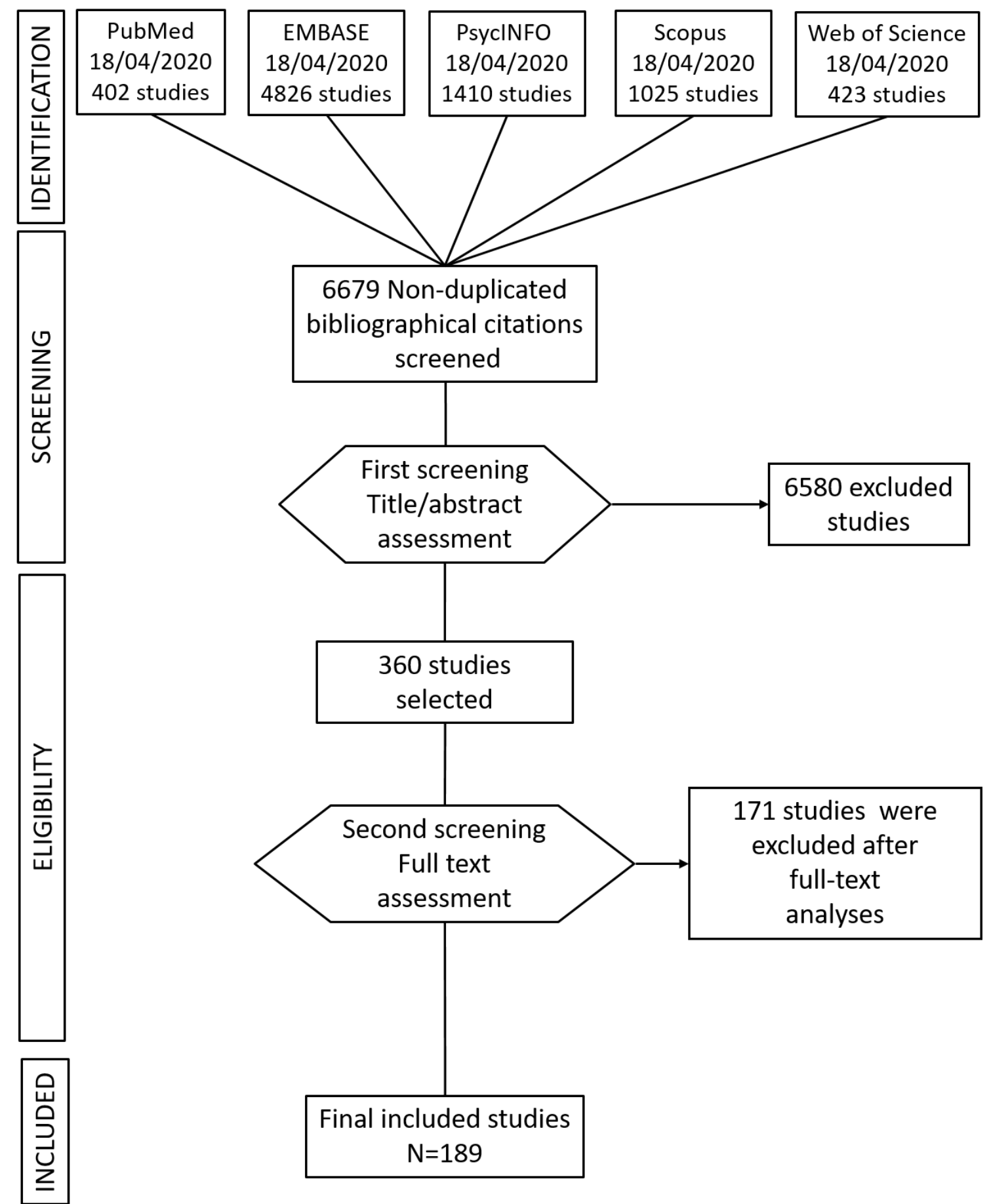




\section{Study characteristics}

The 183 selected studies were inspected in detail. In specific, among the analyzed studies 105 studies were conducted in healthy subjects. The other 78 studies involved clinical populations with some mental illness condition (see Table 2) and 11 of those used healthy subjects as a control group (see Table 2). The clinical populations involved included: amputee patients (20 studies), patients presenting functional motor symptoms (1 study), patients with facial nerve injury (1 study), patients with neurological disorders (26 studies), patients with pain disorders (7 studies); patients with psychiatric disorders (19 studies), patients with psychological disorders (4 studies), and patients presenting other clinical conditions ( 3 studies). For more details see table 2 in which (1) type of participants; (2) mental illness condition; (3) sample size; (4) type of body illusion; (5) control condition; (6) type of multisensory correlation; (7) BI assessment; and (8) mental health related responses, are shown.

\section{BIs for mental health}

The results from the analyzed studies are divided by mental health related responses obtained among the studies in both healthy and clinical populations (see table 3). To summarize the type of BI in healthy and clinical populations table 3 also dived the studies in those that use BIs toward a body part, kinaesthetic body illusions, and FBIs. Moreover, to highlight the effectiveness of BIs in clinical populations a description of the percentage of effectiveness when using BIs for clinical proposes will be provided in figure 2A. Moreover, a graphical representation of the different types of BIs used in clinical populations will be shown in figure $2 \mathrm{~B}$.

\section{BIs to modulate motor responses}

\section{Evidence from healthy subjects}

Twelve studies used BIs towards body parts through the MBI, the RHI, or virtual ownership illusions (T Dummer et al., 2009; Grunt et al., 2017; Hadoush et al., 2013; Heed et al., 2011; Holmes et al., 2006; Kong et al., 2017; Newport et al., 2010; Pyasik et al., 2019; Rjosk et al., 2016, 2017; Schütz-Bosbach et al., 2009, 2006). One study used kinaesthetic illusions (Tidoni et al., 2014), and three studies used FBOI towards virtual bodies (Banakou \& Slater, 2014; Burin et al., 2019; Kokkinara et al., 2016). These studies demonstrated that BOIs induced in controlled experimental setups are able to elicit different motor related responses. (1) BOIs can induce the sense of agency toward a fake body part (Kong et al., 2017; Newport et al., 2010; Schütz-Bosbach et al., 2006, 2009). In specific, Kong and colleagues (2017) demonstrated that a virtual hand illusion (VHI) induced through synchronous VTS can elicit a sense of agency similar in intensity to that experienced for real hand actions in a real context. Newport and co-authors (2010) used the supernumerary limb illusion though synchronous VTS and VMC, and observed that even though sense of ownership can be induced toward more than one fake limb, sense of agency can 
be induced only toward one fake limb. In their studies, Schütz-Bosbach et al. $(2006,2009)$ used the RHI paradigm to demonstrate that observation of motor actions linked to the self through a fake limb led to longer periods of sensorimotor cortical inhibition (no facilitation of brain's motor system) compared to the observation of a static fake hand, but the opposite effect occurred (facilitation of brain's motor system) when observing identical motor actions attributed to another person. (2) Nevertheless, others found that BIs elicited by synchronous VMC through a mirror can improve motor performance associated with functional motor learning-related to changes on brain plasticity, as shown by Rjosk et al. $(2016,2017)$ in a MBI. (3) BOIs can affect the neural processing of action: when altering the perceived size of the hand within a RHI paradigm, a recalibration of the hand position that affect reach-grasp-actions is observed (Heed et al., 2011). (4) BOIs can activate cortical networks related to motor control such as the primary motor cortex (Grunt et al., 2017; Hadoush et al., 2013), and the primary sensoriomotor cortex (Rjosk et al., 2017) through the MBI; (5) BOIs modulate motor actions and can induce a perceptual perception of movement in absence of physical movement (Burin et al., 2019; Timothy Dummer et al., 2009; Holmes et al., 2006; Tidoni et al., 2014). Specifically, Burin et al. (2019) used a FBOI towards a virtual body while introducing visual manipulations of the performed action when participants has a 1PP over the virtual body in comparison to a 3PP. Dummer and co-authors (2019), used the RHI to demonstrate synchronous VMC provide stronger perception of movement compared to asynchronous VMC. In their study, Holmes and colleagues (2006) conducted 5 different experiments to investigate the effects of visual exposure to a real hand, a rubber hand, or a wooden block on reaching movements made with the unseen left hand occluded with a parasagittal mirror; they found that the mere exposure to incongruent visual and proprioceptive information about the reaching hand location have an impact on reaching movements independently on whether an ownership illusion was concurrently experienced. (6) By inducing the RHI in 20 expert pianists, Pyasik et al. ( 2019) demonstrated that increased amount of motor-related afferent and efferent signals does affect the construction and the coherence of body ownership, thus showing the role of movements in the process of body ownership. In addition, it is possible to create the illusion of performing some specific motor actions through virtual FBOI, as in the case of the speaking illusion induced through lips synchronous VMC (Banakou \& Slater, 2014), and of the walking illusion in which a 1PP view of a walking avatar could elicit the illusion of walking in seated participants (Kokkinara et al., 2016).

Evidence from healthy subjects demonstrate that is possible to induce changes in motor responses by manipulating the internal body representation through the use of different types of BOIs. In this regard, the visuo-proprioceptive conflict recalibrates perceived position and therefore has an impact on motor planning. Similarly, an incongruent visuomotor mapping experiment induces automatic motor compensations to cope with specific goals, as in goal directed actions, or to 
compensate the conflict, as in the case of the line-and-circle. Such results paved the way to attempt to use $\mathrm{BI}$ in clinical populations presenting motor disorders.

\section{Results in clinical populations}

All the seventeen analyzed studies directed to modulate motor responses in clinical populations used BI toward a body part (see Table 3) (Cha \& Oh, 2016; Cristina et al., 2015; Diers et al., 2010; Dohle et al., 2009; Grunt et al., 2017; Ji \& Kim, 2014; J. A. Kang et al., 2017; Kim \& Lee, 2015; Kojima et al., 2014; Lin et al., 2014; Louw et al., 2017; Paolucci et al., 2020; Perepelkina et al., 2019; Spychala et al., 2020; Thieme et al., 2013; Weber et al., 2019; Wu et al., 2013). Among the seventeen studies, fourteen studies used the MBI to induce BOI toward a body part. More specifically, in two studies, using the MBI paradigm, patients observed orofacial movements in a tablet screen, compared to a conventional facial mime rehabilitation training in patients suffering from facial paresis, showing that facial movement was significantly larger in the tablet MBI group (J. A. Kang et al., 2017; Paolucci et al., 2020). In the study from Paolucci and colleagues (2020), the authors conducted a double blind randomized control trial with twentytwo patients presenting peripheral facial nerve palsy, to test a neurocognitive-rehabilitative approach through mirror-therapy and motor-imagery, integrated into the traditional rehabilitation with mime-therapy and myofascial-approach. The study from Kang and co-authors (2017) investigated the impact of mirror therapy using a tablet PC for patients with post-stroke central facial paresis in a prospective randomized controlled study. Among the fourteen studies, nine studies were directed to investigate the effectiveness of the MBI in upper limb motor recovery (Cristina et al., 2015; Diers et al., 2010; Dohle et al., 2009; Grunt et al., 2017; Kim \& Lee, 2015; Kojima et al., 2014; Kumar et al., 2016; Louw et al., 2017; Thieme et al., 2013). Six studies investigated the MBI for upper limb motor recovery in patients with stroke (Cristina et al., 2015; Dohle et al., 2009; Kim \& Lee, 2015; Kojima et al., 2014; Thieme et al., 2013; Wu et al., 2013), one with patients with pain shoulder (Louw et al., 2017), and another one used amputee patients with phantom limb pain (Diers et al., 2010). In the studies conducted by Cristina et al., 2015, and the study by Dohle and colleagues (2019), the authors compared the effects of MBI in a group of patients with stroke suffering from upper limb paresis, in comparison with the conventional training treatment, showing that MBI resulted more for motor recovery. Kim and Lee (2015) investigated the effects of the MBI in combination with biofeedback functional electrical stimulation (BF-FES) on motor recovery of the upper limb after stroke that resulted in a feasible and effective method for motor recovery of the upper limb after stroke compared to the conventional rehabilitation training. Kojima and co-authors (2014) demonstrated that a combined treatment of electromyography-triggered neuromuscular stimulation combined with the MBI might be as effective as independent MBI in patients with stroke. In the study from Thieme and colleagues (2013), the authors showed no effect on sensorimotor function of the arm, activities of daily living and quality of life of mirror therapy compared to a control intervention after stroke. 
Nevertheless, a positive effect on visuospatial neglect was observed. Wu and co-authors (2013), compared the effects of the MBI versus a control training for movement performance, motor control, sensory recovery, and performance of activities of daily living in people with chronic stroke, and found that even though the application of the MBI after stroke might result in beneficial effects on movement performance, motor control, and temperature sense, this was not translated into daily functions in patients with chronic stroke.

Three studies used MBI to improve balance and walking ability in patients with stroke (Cha \& Oh, 2016; Ji \& Kim, 2014; Lin et al., 2014). In both, the studies from Cha and Oh (2016), and the study from Ji and Kim (2014) the MBI has been resulted effective to facilitate the effects of a task-oriented exercise on balance function of patients with post stroke hemiparesis compared to a conventional training group. Moreover, in the study from Lin and colleagues (2014), the authors investigated the effects of Mesh Glove $(\mathrm{MG})+\mathrm{MBI}$, compared to a MBI alone, and a conventional training group in 43 patients with stroke and shown that while MBI + MG improved manual dexterity and ambulation. MBI + MG and MBI alone reduced motor impairment and synergistic shoulder abduction more than conventional training group.

In the study by Spychala and colleagues (2020), similarly to the RHI the authors used an embodiable, anthropomorphic robotic hand, which was integrated into a closed-looped EEGbased brain-computer interface (BCI) to resemble the content of the mental imagery action (hand flexion-extension) as closely as possible in nine patients with stroke in a chronic phase with arm paresis compared to a healthy control group. The authors found that most individuals integrated the robotic hand into their body scheme. With respect to robot hand control, none of the participants achieved a high level of control, but most managed to control the robot hand actions to some degree. Importantly, patients and healthy controls achieved similar performance levels. Others used the RHI paradigm and a visual-kinaesthetic VHI to investigate multisensory bodily illusion dynamics in patients with somatoform disorders (Perepelkina et al., 2019), compared to healthy control group and observed that one of the reasons for persistent symptom perception in patients with somatoform disorders is the rigidity of multisensory stimuli perception, at least in the visual-motor domain. Moreover, an immersive virtual reality mirror therapy was tested to improve upper limb paresis in patients with stroke, showing a small improvement in motor outcomes (Weber et al., 2019).

\section{BI to modulate pain responses}

\section{Evidence from healthy}

Twelve studies used BIs to better understand how BOIs can modulate pain perception in healthy subjects (see table 3). Specifically, fourteen studies used BOIs toward body parts (Capelari et al., 2009; Christoffersen et al., 2012; Coleshill et al., 2017; Fang et al., 2019; M. Martini et al., 2013; 
Matteo Martini et al., 2015; Nierula et al., 2017), and two studies used FBOIs (Hänsel et al., 2011; Matteo Martini et al., 2014). Among the studies that used BOI toward a body part, three studies used the RHI paradigm (Capelari et al., 2009; Coleshill et al., 2017; Fang et al., 2019). The study by Capelari and co-authors (2009), use the RHI paradigm to investigate whether different types of tactile stimulation (touch pain stimulus and asynchronous VTS) influence the RHI and found that tactile-painful stimuli could induce the same illusion as the usual tactile stimuli. Moreover, the authors found that that the localisation of pain may also be distorted by manipulated visual cues. Coleshill and colleagues (2017) shown that it is possible to induce pain perception in a fake rubber arm when applying thermal pain stimulus and synchronous VTS. Furthermore, the authors also shown that by applying a sham analgesic stimulus to the rubber arm it is possible to significantly decrease the severity of reported pain in healthy subjects. Fang and colleagues (2019) found that the using the RHI paradigm is possible to attenuate perceived pain, through and experimental non-contact infrared laser, intensity and unpleasantness, and that such analgesic effect is positively correlated with the RHI. Four studies used the VHI, three of them used full virtual bodies to induce the illusion (M. Martini et al., 2013; Matteo Martini et al., 2015; Nierula et al., 2017), and one used augmented virtual reality (Christoffersen et al., 2012). Specifically, in the studies conducted by Martini and co-authors 2013, 2015 the authors demonstrated that by changing the morphological characteristics of the virtual arm through an embodied blue virtual arm, or through a transparent virtual arm is possible to induce analgesic responses to experimentally induced pain through thermal stimuli of increasing temperature. Nierula and colleagues (2017) demonstrated it is possible to induce analgesic responses to an experimental pain stimulus when seeing the virtual arm in exactly the same location as the real one. In the study conducted by Christoffersen and colleagues (2012), the authors showed that by changing the size (enlarged vs. decreased) of the observed virtual arm it is possible to modulate pain perception, and that the sense of control over the observed hand also influences pain perception. Moreover, it has been shown that when identifying with a full virtual body through synchronous VTS is possible to decrease experimental pain perception in healthy subjects, showing an interaction between the vision of the body and pain perception (Hänsel et al., 2011; Matteo Martini et al., 2014).

Overall, the results from healthy subjects provide empirical support for the relationship between body ownership and pain perception, which may advance our understanding of the mechanisms of body pain and promote pain rehabilitation for clinical applications.

\section{Results in clinical populations}

Twenty-four studies used BIs to reduce pain perception in clinical populations (Alphonso et al., 2012; Anaforoğlu Külünkoğlu et al., 2019; Chan et al., 2019; Choi et al., 2019; Darnall \& Li, 2012; Diers et al., 2010; Finn et al., 2017; Giummarra, Fitzgibbon, et al., 2010; Hwang et al., 
2014; Kazemi et al., 2015; Lopez-Carballo et al., 2018; Louw et al., 2017; Matamala-gomez et al., 2018; Ol et al., 2018; Ortiz-Catalan et al., 2016; Osumi et al., 2019; Pamment \& Aspell, 2017; Ramadugu et al., 2017; Reinersmann et al., 2013; Rothgangel et al., 2018; Themelis \& Newport, 2018; Tilak et al., 2016; Tung et al., 2014; Yildirim \& Kanan, 2016) (see table 3). Particularly, twelve studies used the MBI in amputee patients suffering from phantom pain limb (Anaforoğlu Külünkoğlu et al., 2019; Chan et al., 2019; Darnall \& Li, 2012; Diers et al., 2010; Finn et al., 2017; Kazemi et al., 2015; Ol et al., 2018; Ramadugu et al., 2017; Rothgangel et al., 2018; Tilak et al., 2016; Tung et al., 2014; Yildirim \& Kanan, 2016). One study used the MBI with patients with chronic stroke to decrease neck pain discomfort (Choi et al., 2019), and one study used the MBI in patients with shoulder pain (Louw et al., 2017). Four studies were randomized control trials comparing the use of MBI with tactile stimulation alone or with a mirror-tactile stimulation training (Ol et al., 2018), with a sensorimotor exercise training (Rothgangel et al., 2018), a mental visualization therapy (Finn et al., 2017), or with a transcutaneous electrical nerve stimulation therapy (Tilak et al., 2016), for phantom pain relief in amputee patients. One randomized crossover study compared the use of the MBI with a conventional therapy for phantom pain relief in amputees (Ramadugu et al., 2017). Two studies conducted pilot experimental studies without control group to investigate the use of the MBI for phantom pain relief (Anaforoğlu Külünkoğlu et al., 2019; Yildirim \& Kanan, 2016). Two pilot studies were directed to investigate the potential neural correlates of the MBI impact on pain relief, through brain responses in volunteers with unilateral leg amputation using functional magnetic resonance imaging (fMRI) during the course of mirror therapy (Chan et al., 2019), and comparing mirroring movements with imagined or executed movements (Diers et al., 2010). Two studies compared the use of the MBI in bilateral amputee patients with an imagined movements intervention (Tung et al., 2014), and with a healthy control group (Kazemi et al., 2015). One study tested the efficacy of a self-delivered home-based mirror therapy for phantom pain relief (Darnall \& Li, 2012). Others compared a graded motor imagery training in a mirror, compared to active movement training to relief shoulder pain (Louw et al., 2017), or compared a gesture recognition mirror training with the conventional mirror therapy for neck pain relief in patients with chronic stroke (Choi et al., 2019). In all twelve studies the use of the MBI resulted effective for pain relief.

Six studies used virtual limbs ownership illusions trough virtual reality or augmented virtual reality systems (Alphonso et al., 2012; Ortiz-Catalan et al., 2016; Osumi et al., 2019; Rothgangel et al., 2018; Solcà et al., 2018; Themelis \& Newport, 2018). Particularly, two studies attempted to integrate the MBI in an immersive virtual reality system in patients suffering from phantom limb pain (Ortiz-Catalan et al., 2016; Rothgangel et al., 2018). One study aimed to investigate whether manipulations in multisensory information (tactile and visual size) over a virtual arm can modulate pain perception in patients with osteoarthritis (Themelis \& Newport, 2018). In this 
study, the authors observed that stretching the hand both inside and outside the virtual environment led to a reduction in subjective pain ratings, while virtual limb stretching led to changes in body perception with no changes in pressure pain threshold in patients with osteoarthritis (Themelis \& Newport, 2018). In their study, Solcà and co-authors (2018) tested a new digital therapy for chronic pain, a heartbeat-enhanced VR, which combines the VHI and the latest research from multisensory body processing relying on cardiac signals in patients with complex regional pain syndrome (CRPS). They showed a reduced pain perception and an improved hand strength compared to a healthy controls. Others attempted the integration of a prosthetic limb through the observation of virtual limb movements for phantom limb pain relief, and found a decrease of the phantom pain limb as the virtual integrated environment sessions increased (Alphonso et al., 2012). Moreover, in the study conducted by Osumi and colleagues (2019), used the VHI using an immersive VR set-up, to test if providing synchronous VMC to amputee patients with phantom limb pain in the generated virtual environment could be effective for pain relief, and found that the VHI rehabilitation resulted effective for phantom limb pain relief PLP associated with distorted phantom limb movement and body representations (e.g., clamping, gnawing), compared with typical neuropathic sensations (shooting, burning, dysesthesia).

Two studies used the RHI (Giummarra, Fitzgibbon, et al., 2010; Reinersmann et al., 2013). The study conducted by Giummarra and co-authors (2010), aimed at investigating synesthetic pain in lower and upper limb amputee patients when the embodied real (MBI) or the rubber hand (RHI) were threatened, or submitted to high-frequency stimulation, and found that there is not only a sensory components but a motor component when experiencing synaesthesia for pain. Reinersmann and colleagues (2013), used the RHI to investigate higher order multisensory integration of body-relevant stimuli using the rubber hand illusion in patients with CRPS and found an intact ability to perceive illusory ownership over a fake limb, confirming that higher order multisensory integration is unaffected in these patients. One study used kinaesthetic illusions through the walking visual illusion in patients with neuropathic pain with an at-home computer-based version of the visual illusion, including gestural control training, which resulted effective for pain relief (Lopez-Carballo et al., 2018).

Three studies used full body illusions for pain relief in clinical populations (Hwang et al., 2014; Matamala-gomez et al., 2018; Pamment \& Aspell, 2017). One study investigated differences in patients with CRPS when performing (1) a mental rehearsal while in a virtual body swapping condition, (2) a mental rehearsal alone, or (3) another control movement condition, and observed that even though pain intensity did not decrease significantly after treatment in all of the groups, body perception disturbance of the affected limb improved significantly in the virtual bodyswapping group (Hwang et al., 2014). In the study conducted by Matamala-Gomez and colleagues 
(2019), the authors investigated whether by changing the morphological characteristics of an embodied virtual arm, in specific size and transparency, it is possible to modulate pain perception in different types of pain clinical populations (patients with peripheral nerve injury (PNI) or patients with (CRPS). The authors found that all virtual reality conditions globally decreased pain ratings, but increasing virtual arm transparency decreased pain in CRPS and did the opposite in PNI (Matamala-gomez et al., 2018). Pamment and Aspell (2017), used full-virtual body illusions to investigate whether patients' experience of chronic pain could be reduced, and shown that pain intensity in patients with chronic pain was reduced by $37 \%$ by an out-of-body-illusions induced through synchronous VTS.

\section{BI to modulate psychological and cognitive responses Evidence from healthy}

Nine studies used BIs to investigate psychological and cognitive responses in healthy subjects. Three studies used BIs toward a body parts (Braithwaite et al., 2017; Maister et al., 2013; Palomo et al., 2018), and six studies used full body ownership illusions (Banakou et al., 2016, 2018; Falconer et al., 2014; Ferrer-Garcia et al., 2017; Hamilton-Giachritsis et al., 2018; Osimo et al., 2015). In the study conducted by Palomo and colleagues, the authors investigated the RHI in three different groups of subjects with different age, and no subjective, behavioural, or physiological differences linked to age were found. Others used the RHI to investigate the role of intersensory biases in the induction of an experimentally body illusion, in subjects predisposed to spontaneous OBEs, compared with another group without OBEs and found that even in asynchronous VTS conditions 'OBEers' subjects experienced a strong RHI (Braithwaite et al., 2017).

It has been shown that by inducing the sense of ownership through synchronous VTS toward a dark rubber hand it is possible to reduce implicit racial bias (Maister et al., 2013). In line with this study, others used a full body ownership illusion toward a white or black virtual body, and showed that implicit racial bias in white participants decreased more after embodying the black virtual body (Banakou et al., 2016). Other investigations used full virtual body illusions through synchronous visuomotor correlations to place the parents in the position of a child and observed that after experiencing the illusion participants with negative maternal behavior increased their levels of empathy toward the child. In addition, the negative maternal behaviour led to increased feelings of fear of violence, and physiological data indicated greater levels of stress (HamiltonGiachritsis et al., 2018). FBOI with virtual bodies has also been used in high self-critical healthy subjects in which the rehearsing the delivery (adult virtual body) and receipt (child virtual body) of compassionate behavior leads to a reduction in self-criticism, both when the illusion is experience from a 1PP and from 3PP (Falconer et al., 2014). Moreover, in the recipient rehearsing a positively increasing self-compassion in self-critical individuals had shown (Falconer et al., 
2014). Moreover, being embodied in a world wild known intelligent character such Einstein lead to a better performance in a virtual cognitive task, compared to being embodied in a normal young virtual body (Banakou et al., 2018). Regarding this, in the study by Ferrer-Garcia and colleagues (2017), the authors demonstrate that by being embodied in a virtual body showing a $40 \%$ larger virtual body is possible to produce body anxiety responses in a non-clinical sample, than while being embodied in a virtual body with the subjects real measurements (Ferrer-Garcia et al., 2017). In the study from Osimo and colleagues (2018), the authors submitted the participants to an alternately switched experience between a virtual body resembling themselves where they described a personal problem, and a virtual body representing Dr Sigmund Freud, from which they offered themselves counselling. Results showed that when the counsellor resembles Freud participants improve their mood more with respect to when the counsellor was their selfrepresentation (Osimo et al., 2015).

\section{Results in clinical populations}

Five studies used BIs to investigate psychological and cognitive responses in clinical populations (Ensink et al., 2016; Falconer et al., 2016; Jalal et al., 2020; Rabellino et al., 2018; Seinfeld et al., 2018). Jalal and co-authors used the RHI paradigm to investigate a "multisensory stimulation therapy" for patients with an obsessive-compulsive disorder, which consisted in contaminating a rubber hand through fake feces after 5 minute of synchronous or asynchronous VTS. The authors showed that either synchronous or asynchronous VTS induced an equally vivid ownership illusion, and both conditions provoked similar contamination reactions of equal magnitude (Jalal et al., 2020). This study paved the way to the use of BIs for exposure and response prevention therapy in patients with obsessive-compulsive disorder (Jalal et al., 2020). The RHI paradigm was also used in the study from Rabellina and collegaues (2018) in which the authors shown that by improving and restoring an embodied sense of agency through the RHI in patients with posttraumatic stress disorder in alteration in the sense of ownership and agency have been shown after the trauma experience (Rabellino et al., 2018).

FBOI through virtual bodies induced by synchronous VMC had been used to improve empathy in offenders by experiencing a virtual situation of domestic violence from the perspective of the victim (Seinfeld et al., 2018). Falconer and co-authors (2016), investigated whether the effects of self-identification with full virtual bodies (child or adult) could be exploited to increase selfcompassion in patients with depression, and found significant reductions in depression severity and self-criticism, as well as to a significant increase in self-compassion, from baseline to 4-week follow-up (Falconer et al., 2016). In the study from Karin and colleagues (2016), the authors tested the mirror paradigm (reflection of the self in a mirror) from Kernenberg \& Normandin (2002, unpublished document), consisting in asking a series of questions that further activate attention to the positive and negative aspects of self as well as aspects that may be dissociated in 
front of a mirror, in children with history of sexual abuse, and in children without any trauma, and found that embodied self-experiences of children with histories of sexual abuse were significantly more negative than those of non-abused controls (Ensink et al., 2016).

\section{BI to modulate body representation}

\section{Evidence from healthy}

Sixty studies used BOIs toward a body part to investigate body representation in healthy subjects. Among the fifty-nine studies, thirty-five studies used BOI toward a body part. Eight studies were directed to investigate how participant's characteristics can influence the ownership illusion when using the RHI paradigm such as age, showing a preserved ability to integrate the perceptual illusion of the RH (Riemer et al., 2019; Zeller \& Hullin, 2018); laterality, which even though some studies did not found differences in experiencing the RHI between left-handers and righthanders (Smit et al., 2017), others found a more vivid illusion when using the RHI in left-handers compared to right-handers (Ocklenburg et al., 2011); psychosis proneness, which foster the sense of ownership toward the rubber hand (Germine et al., 2013); non-clinical autism spectrum that could influence the perception of the rubber arm position (Palmer et al., 2013); or participants presenting some medically unexplained symptom, which presented lower RHI experience compared to a normal participants (Miles et al., 2011). Six studies aimed at investigating how by the manipulation of body sensory or cognitive components is possible to modulate the ownership illusion toward a body part (Dempsey-Jones \& Kritikos, 2014; Ferrè et al., 2015; Lopez et al., 2010; Morgan et al., 2011; Ponzo et al., 2018; Schomaker et al., 2011). More specifically, three studies manipulated vestibular information in healthy subjects while performing the RHI paradigm (Ferrè et al., 2015; Lopez et al., 2010; Macauda et al., 2015; Ponzo et al., 2018), and one study during the VHI (Schomaker et al., 2011), and found that through the vestibular stimulation participant increased the sense of proprioceptive drift toward the fake body part enhancing the sense of ownership. One study investigated the effect of ketamine on the experience of illusory ownership toward the rubber hand, which mimics the perturbed sense of body ownership seen in schizophrenia enhancing the possibility to induce body illusions (Morgan et al., 2011). Dempsey-Jones and co-authors, observed that through high-order cognitive factors it is possible to modulate subjective (sense of embodiment), but not proprioceptive components (proprioceptive drift) of self-representation (Dempsey-Jones \& Kritikos, 2014).

Twenty studies used BOI toward a body part to understand how body representation is malleable and can be easily distorted during BIs. Particularly, two studies investigated the possibility to induce the illusion of owning a third arm by applying synchronous VTS when the rubber hand is close to the real hand (Folegatti et al., 2012; Guterstam et al., 2011), showing that the body representation can easily be updated to incorporate an additional limb. Other demonstrated that it is possible to induce the illusion of owning an elongated rubber hand illusion through synchronous 
VTS (Schaefer et al., 2007), and an elongated virtual arm illusion (Kilteni et al., 2012). Others demonstrate that it is possible to induce independent ownership and control over a third rubber hand without loss of ownership in the real hands (Bashford \& Mehring, 2016). In the study by Foell and colleagues (2013), the authors shown that through asynchronous VMC when using the MBI it is possible to modulate the perceived body integrity, in healthy subjects, inducing the feeling of owning additional limbs (Foell et al., 2013). Moreover, by applying asynchronous VTS over an embodied virtual arm it is possible to recalibrate the metrics of the stimulated limb towards elongation (Perez-Marcos et al., 2018). Moreover, in another study, by removing visual feedback from a virtual hand and arm participants felt a proximal retraction of their limb, resembling the telescoping experienced by phantom limb patients (Thøgersen et al., 2018).

Changes in the rubber hand position can be perceived as self-generated in the RHI (Abdulkarim \& Ehrsson, 2016), as well as the movement of the RHI inducing a dissociation of ownership and agency from the real body part to the fake body part (Kalckert \& Ehrsson, 2012; Louzolo et al., 2015). Nine studies investigated the perceptual components of body representation when using BI through the RHI (David et al., 2014; Haggard \& Jundi, 2009; Limanowski \& Blankenburg, 2016; Mulvey et al., 2012; Mussap \& Salton, 2006; Ocklenburg et al., 2012; Riemer et al., 2014), the MBI (Romano et al., 2013), and through the enfacement illusion (Bufalari et al., 2014). In addition, two studies used kinaesthetic illusions by applying a simultaneous vibration over antagonistic biceps and triceps muscle tendons, and observed an altered representation of the body in which participants experiences a perceived telescoping effect of the arm towards the elbow (de Vignemont et al., 2005; Longo et al., 2009).

Sixteen studies used FBOI to investigate changes in body representation in healthy subjects. More specifically, eight studies investigated the possibility of feeling embodied in another body presenting different morphological characteristics such as feeling embodied in a Barbie doll body by inducing a body swapping illusion through a HMD in healthy subjects with high interpersonal factors of schizotypy (Van Doorn et al., 2018). In addition, later investigations using the Barbie doll illusion observed that by changing the size of the fake body affected how participants perceived the world, perceiving the surrounded objects larger or smaller depending of the body size (van der Hoort et al., 2011). Similarly, being embodied in a virtual child body may affects the perceived size of the surrounded environment as well as generate implicit changes in attitude (Banakou et al., 2013). The body swapping illusion had been also used to induce embodiment into a full fake body presenting different sizes (slim or obese body) that provoked changes directly related with body satisfaction, specifically in women (Preston \& Ehrsson, 2014; Preston \& Henrik Ehrsson, 2018). Moreover, the possibility to induce the illusion of owning a large belly size through full virtual body illusion had been shown by Normand and co-authors (Normand et al., 
2011), as well as the related body dissatisfaction responses when owning an obese body (Preston \& Ehrsson, 2016).

In addition to the modulation of body satisfaction by using FBOI, another studyusing a virtual body swapping illusion, in which participants observed the virtual body with different sizes from a 1PP (egocentric frame) or 3PP (allocentric frame), showed that there are two different mechanisms underlying body image disturbances: one related to real-time perception-driven inputs (egocentric frame), and one related to abstract knowledge, beliefs, and attitudes related to a person's body (allocentric frame) (Corno et al., 2018). Serino and co-authors also demonstrate that it is possible to induce a change in the memory of the body through a VR body swapping technique (S. Serino et al., 2016). Moreover, being embodied in full virtual child body can modulate the subsequent real speaking (Tajadura-Jiménez et al., 2017). Five studies investigated the influence of a full virtual body illusion from a 1PP: (1) to induce real body postural discomfort (Bergström et al., 2016); (2) to stimulate some motor responses such as the walking illusion (Kokkinara et al., 2016); (3) for motor errors monitoring (Pavone et al., 2016; Valzolgher et al., 2018); or (4) to incorporate robotic hands into the own body through synchronous VMC controlled by a brain machine interface (Alimardani et al., 2013).

Serino and colleagues (2018) observed that there are age related changes when perceiving a virtual FBOI, where participants between 26-to-55-year-old were more resistant to changes induced by the bodily illusion, compared to a young group of people (19-to-25 years old) (S. Serino et al., 2018).

Overall, results from studies with healthy subjects show that it is possible to use BOIs to better understand the neural correlations of body representation in the brain, and to induce body distortions that are present in clinical populations for improving clinical care when dealing with such body distortions.

\section{Results in clinical populations}

Thirty-four studies used BIs to improve body representation in clinical populations. Eight studies used BIs toward a body part in patients with schizophrenia (Ferri et al., 2014; Ferroni et al., 2019; Lev-Ari et al., 2015; Peled et al., 2003; Prikken et al., 2019; Sandsten et al., 2020; K.N. Thakkar et al., 2011; Van Haren et al., 2018). Six studies of those used the RHI (Ferri et al., 2014; LevAri et al., 2015; Peled et al., 2003; Prikken et al., 2019; K.N. Thakkar et al., 2011; Van Haren et al., 2018). These studies demonstrated that patients with schizophrenia are more sensitive to the RHI due to abnormalities in temporo-parietal networks that have been associated with a more flexibly representation of the self-body in the brain (K.N. Thakkar et al., 2011). However, it is shown that strength of the illusion and the ability to integrate the body illusion over time was 
inconsistent in patients with schizophrenia compared to healthy subjects because of their cognitive impairment (Lev-Ari et al., 2015).

Two studies used the enfacement illusion (EI) (Ferroni et al., 2019; Sandsten et al., 2020). In the study conducted by Sandsten and colleagues (2020), healthy subject and patients with schizophrenia were exposed to a video showing a movie of the face of an "other" individual looking straight into the camera while receiving synchronous VTS on the face, or were alternative exposed to a movie of their self-face without tactile stimulus; results showed deviations in specular self-recognition in patients with schizophrenia compared to healthy controls. In the study by Ferroni and co-authors (2019), the authors used the enfacement illusion in both patients with schizophrenia and healthy subjects and showed that this illusion is not only confined to self-sphere but it also affects the way subjects discriminate others, representing a potential crucial aspect in the social domain in patients with schizophrenia.

The RHI was also adopted to investigate differences in the influence of the preceding sensory context on motor function, in subjects with clinical and subclinical features of autism spectrum disorder, and found that despite both categories of individuals experienced the perceptual effects of the illusion, the impact of the illusion on in subsequent reaching movements was limited with respect to healthy subjects(Palmer et al., 2015).

Seven studies used BIs toward a body part to investigate alterations in body representation in patients with eating disorders (ED) compared to a healthy control group (Carey \& Preston, 2019; E. Eshkevari et al., 2012; Ertimiss Eshkevari et al., 2014; Keizer et al., 2014; Tagini et al., 2020; Zopf et al., 2016). Specifically, a significant reduction in hand size estimation and low levels of both implicit and explicit body satisfactions have been shown when using the moving rubber hand illusion (Carey \& Preston, 2019). However, when using the conventional RHI a stronger experience of ownership over the rubber hand in patients with anorexia nervosa (AN) has been shown, indicating a more malleable body representation in AN patients compared to healthy females (E. Eshkevari et al., 2012; Keizer et al., 2014). Moreover, another study using the RHI paradigm demonstrate that such body representation malleability in patients with AN persists to a large degree following weight regain (Ertimiss Eshkevari et al., 2014). In this study the authors compared the sense of ownership toward the RH between a group of recovered patients with ED, a group suffering from ED, and a healthy control group, and observed that females in the recovered group presented similar results to the ED group. In their study, Zopf and colleagues (2016) used the RHI to test whether body localization abilities are altered in patients with AN compared to a healthy control group: patients with AN displayed a compromised proprioceptive encoding of hand location (Zopf et al., 2016). In addition, Tagini and collegaues (2020), used the VHI in participants affected by obesity and participants with a healthy weight, to observe a 
possible unusual susceptibility to the RHI related to anomalous bodily self-experience, and found that even though participants with obesity reported a strong ownership illusion, a reduction in recoding self-location was also observed (Tagini et al., 2020).

Two studies used FBOIs in patients with ED (Forghieri et al., 2016; Keizer et al., 2016). Particularly, Keizer and colleagues (2016), used a full virtual body illusion to investigate whether it is possible to modulate body size estimation of real body parts that are more emotionally salient than the hand (e.g. shoulders, abdomen, hips) in patients with AN. They found that it is possible to decrease the overestimation of body size, especially of emotionally body parts, with effects remaining at least up to $\sim 2$ hours and 45 minutes after the illusion was induced (Keizer et al., 2016). Moreover, others used a FBOI through a mirror by showing the participants image in a mirror and then the image of a fashion model representing their body ideal in comparison to a neutral body stimuli: patients with ED showed higher levels of body dissatisfaction by showing higher levels of postural destabilisation than healthy controls, when facing their self-reflection or that of a fashion model (Forghieri et al., 2016). This study, demonstrated the effectiveness of such mirror FBOI paradigm in assessing body dissatisfaction in patients with ED.

Three studies used BOI toward a body part in patients with an amputation (D'Alonzo et al., 2015; Giummarra, Georgiou-Karistianis, et al., 2010; Imaizumi et al., 2017). Guimmarra and colleagues (2010), showed that it is possible to induce embodiment toward a rubber or real passive hand reflected in a mirror while applying different sensory stimulations (touch, pressure, movement, vibration, and temperature) in patients with upper limb amputation (Giummarra, GeorgiouKaristianis, et al., 2010). The RHI was also used to investigate whether it is possible to induce the sense of ownership toward the rubber hand by applying synchronous vibrotactile stimulation over the stump in patients with upper limb amputation, and patients reported feeling of ownership toward the rubber hand after 90 seconds of synchronous vibrotactile stimulation (D'Alonzo et al., 2015). Imaizumi and colleagues instead showed that it is possible to enhance the sense of agency toward the phantom limb after 15 minutes of performing the MBI in patients with a upper limb amputation (Imaizumi et al., 2017)..

Twelve studies used BOI toward a body part to investigate body representation in patients with neurological disorders (Bolognini et al., 2014; Demartini et al., 2016; Fiorio et al., 2014; Fossataro et al., 2018; Garbarini et al., 2015; Jenkinson et al., 2013; Lenggenhager et al., 2012, 2015; Martinaud et al., 2017; Nava et al., 2014; Ng \& Singh, 2015; Rae et al., 2018). Particularly, a study using the RHI in patients with functional motor symptoms demonstrated that the sense of body ownership is not impaired these patients (Demartini et al., 2016). In the study by Rae and co-authors (2018), the RHI paradigm was testes in patients with Tourette Syndrome which presented alterations in visuo-tactile integration and observed that ownership illusion towards the 
RHI contributes to susceptibility to the premonitory sensations that are a precipitating factor in tics (Rae et al., 2018). Participants with Tourette Syndrome shown less proprioceptive drift towards a rubber hand, but strong feelings of embodiment compared to healthy control group, that were positively correlated with premonitory sensation severity in Tourette Syndrome (Rae et al., 2018). An alien hand illusion was used in the study by Fossataro and colleagues (2018), to investigate whether in patients with damages of cerebrovascular origin, who experience a pathological embodiment (E+) (sense of embodiment toward other people body parts) with spared tactile sensibility, a coherent body ownership could be restored by introducing a multisensory conflict between what the patients feel on the own hand and what they see on the alien hand. The authors reported that in patients experiencing pathological embodiment, an incongruent VTS of the own and of the alien hand reduces the delusional body ownership over the alien hand, by restoring the access to the perceptual self-identity system (Fossataro et al., 2018). These results were previously found after performing a tool-use training in patients with brain damage who manifested a pathological embodiment of other people body parts (Garbarini et al., 2015). In the study from Martinaud and colleagues (2017) the authors used the RHI in patients with right hemisphere lesions due to an ischaemic or haemorrhagic stroke, and found that participants may experience feelings of ownership over a rubber hand by 'visual capture or co-location', without any tactile stimulation. The 'visual capture of ownership' was found to be associated with proprioceptive deficits and lesions mostly in the frontal operculum and the premotor motor cortex after stroke (Martinaud et al., 2017). In addition, in their study Bolognini and co-authors showed that it is possible to decrease somatoparaphrenia in brain damage patients by applying synchronous VTS in the RHI (Bolognini et al., 2014). This results had been previously shown when using synchronous VMC in the MBI in patients presenting somatoparaphrenia because of a stroke injury (Jenkinson et al., 2013). .. An adaptation of the RHI was used in patients with xenomelia (the foreign limb syndrome), with the 'rubber foot illusion': patients experienced ownership illusion over the undesired limb similar to the control healthy group, and the vividness of the illusion correlated positively with the strength of amputation desire (Lenggenhager et al., 2015). Moreover, the use of the MBI have been used in patients with unilateral neglect during 4 weeks and observed that the MBI is a simple treatment that effectively improves symptoms in these patients (Ng \& Singh, 2015).

A study conducted in patients with spinal cord injury (SCI) found that, even though no alteration had been found related to the illusory ownership in the RHI , the associated proprioceptive drift typically observed in healthy subject was missing (Lenggenhager et al., 2012). In the study by Nava and colleagues, congenital blind subjects and late blind subjects were exposed to the experimental paradigms of the RHI and the Aristotle illusion, which is a perceptual disjunction consisting in crossing the fingers while touching a small and spherical object, and that results in 
the feeling like you are touching two spherical objects instead of one (Nava et al., 2014). The study demonstrated that the while the RHI did not occur in congenital blind adults, late blind individuals showed proprioceptive and auditory drifts towards the rubber hand even though the illusions was not subjectively reported congenitally and late blind individuals also did experience the Aristotle illusion (Nava et al., 2014).

\section{BI to modulate physiological responses}

\section{Evidence from healthy}

Fifteen studies used BOIs in healthy subjects to investigate physiological, sensory and neurobiological responses. Among these studies, twelve studies induced BOIs toward a body part (Barnsley et al., 2011; Bekrater-Bodmann et al., 2012; Evans \& Blanke, 2013; Guterstam et al., 2013; Johnson et al., 2016; Kilteni et al., 2016; Kilteni \& Ehrsson, 2017; Miller et al., 2017; Moseley, Olthof, et al., 2008; Perez-Marcos et al., 2009; Tieri et al., 2017). Five studies used the RHI (Barnsley et al., 2011; Bekrater-Bodmann et al., 2012; Johnson et al., 2016; Kilteni \& Ehrsson, 2017; Moseley, Olthof, et al., 2008). Particularly, in the study by Moseley and colleagues (2008), the authors investigated the effect of undergoing a RHI illusion on the skin temperature of the "substituted" hand, showing that the RHI evokes a limb-specific decrease in the temperature of the participant's own hand and a decrease in the weight given to tactile information from that hand. Furthermore, the magnitude of both effects correlates with the strength of the illusion (Moseley, Olthof, et al., 2008). At a physiological level, Barnsley and colleagues (2011), demonstrated in healthy subjects undergoing a RHI the histamine reactivity of the substituted hand increases, "as though the actual arm is being 'rejected"'.(Barnsley et al., 2011). In their study Bekrater and co-authors demonstrated that the vivideness of the $\mathrm{RHI}$ and corresponding neural processes are stable across long periods of time (of the order of several months). In an fMRI study the authors demonstrated that there is an individual degree of susceptibility which prevails in different contexts (horizontal and vertical positions), and an intra-individual stability over time (6 months) with no changes in illusory experience, and that the neuronal processes underlying the RHI (ventral premotor cortex processing) was temporally stable (Bekrater-Bodmann et al., 2012).

Johnson and colleagues (2016), aimed to investigate the skin conductance response, self-reported anxiety and the incidence, type and location of sensations when a perceptually embodied rubber hand was exposed to threatening and non-threatening stimuli (needle vs. brush stimuli), and showed that half of the participants experienced more intense sensations when a perceptually embodied rubber hand was approached by a threatening stimulus i.e. the needle.

Kilteni and Ehrsson (2017), combined the RHI and the force-matching paradigm, which allows psychophysical quantification of somatosensory attenuation to investigate how body ownership determines the attenuation of self-generated touch. In this study the authors found that a rubber 
right hand pressing on the left index finger produced somatosensory attenuation but only when the tactile stimulation was perceived as self-generated (illusory self-touch) (Kilteni \& Ehrsson, 2017). In the study conducted by Miller and colleagues (2017), the authors demonstrated that a visual illusion of tool use recalibrates tactile perception and strongly suggest that visual signals of tool use are a driving signal for embodiment, when using the MBI. Three studies used the VHI (Evans \& Blanke, 2013; Perez-Marcos et al., 2009; Tieri et al., 2017).

Tieri and colleagues (2017) demonstrated that the VHI caused high sense of embodiment toward the $\mathrm{VH}$ and low temperature changes in the real hand with respect to the other experimental conditions, the wooden hand illusion and the discontinuous hand illusion, in which participants experienced high temperature changes. Other used the VHI to demonstrate that there are common neural networks between brain networks activations when inducing illusory hand ownership in virtual reality, and brain mechanisms when performing motor imagery in the fronto-parietal cortex (Evans \& Blanke, 2013; Perez-Marcos et al., 2009). In addition, in the study from Kilteni and colleagues (2016), shown a decrease in the motor evoked potentials amplitudes of the extensor digitorum communis muscle for the contralateral sensoriomotor cortex when embodying participants in a virtual body inducing an illusory missing arm illusion in comparison with an intact virtual arm illusion.

Guterstam and co-authors (2013), shown in four different experiments that it is possible to induce the illusion of having an invisible hand depending on visuotactile-proprioceptive integration that obeys key spatial and temporal multisensory rules confined to near-personal space. Further, they also shown that a stronger feeling of having an invisible hand is associated with a higher degree of effective connectivity between the intraparietal and ventral premotor cortices (Guterstam et al., 2013).

Three studies used FBOI to investigate physiological, sensory or neurobiological responses in healthy subjects (Bergouignan et al., 2014; Llobera et al., 2013; Salomon et al., 2013). In the study from Bergouignan and colleagues (2014), the authors used the body-swapping illusion from the real body position (in body perspective), compared to a $180^{\circ}$ (front perspective) or $30^{\circ}$ (side perspective) body position, to investigate the role of perceiving the world from an in body perspective for the successful episodic encoding of real-life events, while participants were involved in a social interaction. The results demonstrated an efficient hippocampus-based episodic-memory encoding activity when participants experienced the in body perspective condition through the HMD while were involved in a social interaction (Bergouignan et al., 2014). Two studies using FBOI with virtual bodies showed a decrease in skin temperature sensitivity when embodied in a full virtual body co-located with the real body (Llobera et al., 2013), and 
when the virtual body was located at a different position of the real body (Salomon et al., 2013) while receiving synchronous VTS.

\section{Results in clinical populations}

Four studies used BOI toward a body part to investigate neurobiological responses in clinical populations (Bogdanov et al., 2012; Chan et al., 2019; Michielsen et al., 2011; Peled et al., 2003). One study compared the exposure to the MBI with or without motor imagery, in healthy and patients with an upper limb amputation to investigate whether the use of the intact hand reactivates the cortical territory formerly devoted to the now missing limb, and shown that intact hand movements increased activity specifically within the former sensorimotor hand territory during all experimental conditions (Bogdanov et al., 2012). Chan and colleagues (2019), measured brain responses in volunteers with unilateral leg amputation using functional magnetic resonance imaging (fMRI) during a four-week training of MBI, and found an atypical responsiveness to the presented limbs illusion through the MBI in the sensorimotor cortex that was reduced throughout the 4 weeks of the MBI training period that was associated with a reduction in phantom limb pain. A study investigated the neural correlates of the MBI for upper limb motor recovery in patients with stroke, and showed changes in neural activity in the precuneus and posterior cingulate cortex areas, in both unimanual or bimanual motor training (Michielsen et al., 2011). In their study Peled and co-authors investigated the somatosensory evoked potentials (SEPs) differences when using the RHI in schizophrenic patients compared to healthy controls, and showed that patients with schizophrenia presented significant alterations in long latency evoked responses during the RHI compared to healthy controls.

\section{The efficacy of BI on clinical populations}

Among the 78 analyzed studies using BOIs in clinical populations, the $88,2 \%$ of the studies used BOI toward a body parts, $9,4 \%$ of the studies used kinaesthetic illusions, and only $2,4 \%$ used FBOI. Furthermore, $65,9 \%$ of the analysed studies showed a positive effectiveness in the use of BOIs to treat clinical mental illness conditions, the $34,1 \%$ showed neither positive or negative effects in the use of BOIs to clinical mental illness conditions, and no studies among the analysed studies presented negative effects when using BOIs to treat clinical mental illness conditions (see figure $2 \mathrm{~A}$ and $\mathrm{B})$.

\section{Figure 2. Percentage of the type of BOIs used in clinical populations and percentage of clinical effectiveness.}


A)

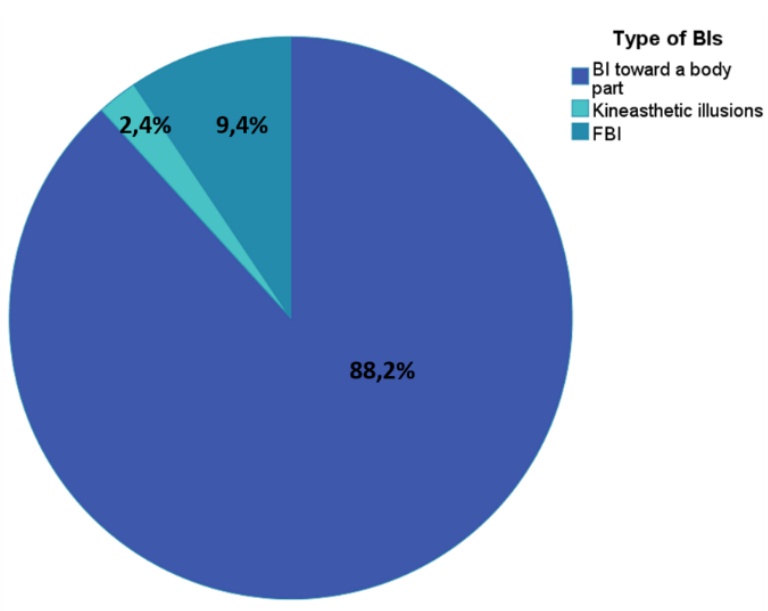

B)

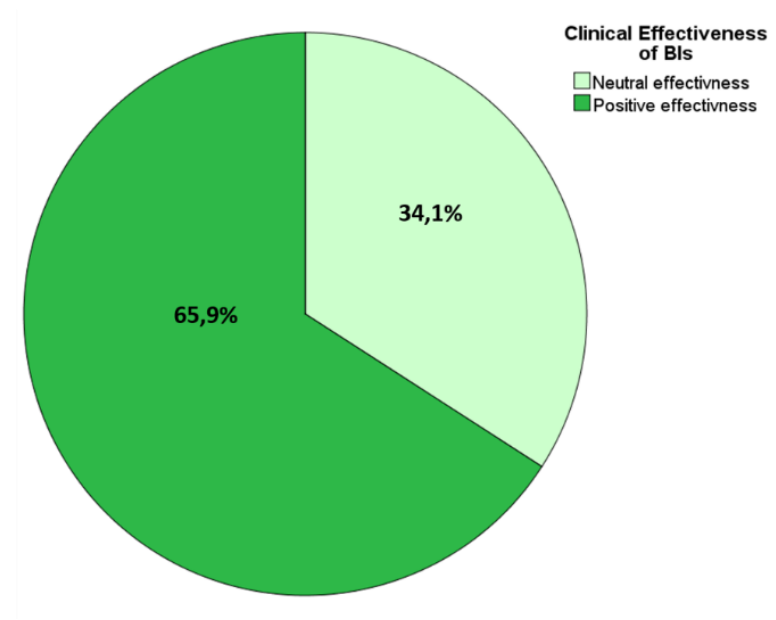

BIs: Body Illusions. FBI: Full-body illusions

\section{Discussion}

The present manuscript aims to provide a systematic review of the use of BIs in the healthy and clinical populations and review how BIs can be adopted to enhance mental health in different mental illness conditions: motor disorders, pain disorders, and psychological and psychiatric amputation, and other neurological disorders. Such disorders tend to present alterations in different clinical responses as in motor responses, pain responses, psychological and cognitive responses, body representation responses, and physiological responses. Following the structure presented in the results sections, results about the use of BIs in clinical populations will be discussed.

\section{BIs to modulate motor responses}

Most of the reviewed studies in this systematic review using BIs to improve motor responses used the MBI (Cha \& Oh, 2016; Choi et al., 2019; Cristina et al., 2015; Diers et al., 2010; Dohle et al., 2009; Grunt et al., 2017; In et al., 2016; Ji \& Kim, 2014; Y. J. Kang et al., 2011; Kim \& Lee, 2015; Kojima et al., 2014; Lin et al., 2014; Paolucci et al., 2020; Thieme et al., 2013; Wu et al., 2013). The MBI has been found useful for motor recovery in many studies (see Bolognini et al., 2015; Ramachandran et al., 2009). Indeed, it has been shown that observing the mirror reflection of own movements increases the excitability of the ipsilateral motor cortex of the moving hand (Garry et al., 2005). In addition, it has also been shown that the mismatch between the performed movement and the observed mirror reflected movement through increases neuronal activity in brain areas associated with self-awareness and spatial attention such as the precuneus cortex, the posterior cingulate cortex, and the posterior parietal cortices (Dohle et al., 2004; Fink et al., 1999; Matthys et al., 2009; Michielsen et al., 2011). 
Following this therapeutically approach others attempted to use the RHI paradigm through a robot hand (Spychala et al., 2020), or through virtual reality with VHI (In et al., 2016; Weber et al., 2019), in which synchronous VMC were provided to activate neuronal activity in the related brain motor areas and induce motor learning. VR setups are particularly important in the context of motor behavior disorders because it offers a tool to control and modulate on-line the motor behavior of a user, through the interplay between motor control loop mechanisms and the effects of embodiment that could drive and "attract" performed movement as in the "follower-effect" (Gonzalez-Franco et al., 2020) or the line-circle experiment (Burin et al., 2019). In the recent study, Matamala-Gomez and co-authors reported the effects of embodiment toward a full virtual body in patients performing a virtual motor rehabilitation training, showing improved motor rehabilitation outcomes in patients with distal radius fracture after five weeks of the immobilization period (Matamala-Gomez, Slater and Sanchez-Vives 2020). However, to date, few studies used virtual embodiment through FBOI to improve motor performance in clinical populations.

\section{BI to modulate pain responses}

The reviewed studies in this systematic review also showed that the MBI had been widely used as an effective BOI toward a body part to reduce pain perception in patients with an amputation (Anaforoğlu Külünkoğlu et al., 2019; Chan et al., 2019; Darnall and Li, 2012; Diers et al., 2010; Finn et al., 2017; Kazemi et al., 2015; Ol et al., 2018; Ramadugu et al., 2017; Rothgangel et al., 2018; Tilak et al., 2016; Tung et al., 2014; Yildirim and Kanan, 2016). Indeed, the first clinical application of the MBI has been for pain relief in patients with phantom pain limb because of an amputation (Ramachandran et al., 1995). In this regard, Melzack (1992) proposes a central role of multisensory integration of bodily signals within the commonly known 'neuromatrix', in the construction of body image and the generation of pain, . Then, the mirror reflection of the healthy limb, which seems visually superimposed to the affected limb in the MBI, generates the illusion of movement of the affected limb allowing the patients relaxing the cramped phantom limb and inducing pain relief. Moreover, in the MBI the correction of the incongruence between the intention to move (motor output) and the visual feedback provided by the mirror reflection of the movement induce relief from phantom limb pain, reactivating the "latent" proprioceptive cortical map of the missing limb (Moseley, Gallace, et al., 2008; Moseley \& Flor, 2012; Ramachandran $\&$ Altschuler, 2009). However, throughout the reviewed studies we found that not always the MBI has been resulted effective in reducing phantom limb pain in amputee patients presenting a telescoped effect (that is, when the distal part of the phantom limb is perceived as shrinking within the stump) (Foell et al., 2014).

Following the 'neuromatrix' approach introduced by Melzack, in this systematic review, we found that other researchers used the RHI for pain relief in chronic pain disorders such as in 
patients with complex regional pain syndrome and patients suffering from synaesthesia (Giummarra, Fitzgibbon, et al., 2010; Reinersmann et al., 2013). The use of the RHI for pain relief in patients suffering from chronic pain has been proposed before by Lotze and Moseley (2007), considering the link between pain and body image distortions again because patients with chronic pain often present body image distortions, disrupting some body-related cortical representations.

Over the last ten years, the development of the technology introduces the use of VR systems to reproduce the RHI in VR but allowing researchers to modify the morphological characteristics of the virtual limb. This specific characteristic could help deal with the link between pain and body image distortions in patients with chronic pain (Matamala-gomez et al., 2019; Matamala-Gomez et al., 2020). In this regard, in this systematic review, some studies used BOI toward body parts through virtual limbs, or virtual FBOI to manage pain perception in patients with chronic pain (Alphonso et al., 2012; Matamala-gomez et al., 2018; Ortiz-Catalan et al., 2016; Osumi et al., 2019; Pamment \& Aspell, 2017).

\section{BI to modulate psychological and cognitive responses}

Through BIs, it is possible to induce a sense of embodiment toward a fake body and, by modify the body's experience, to modify one's mind (Maister et al., 2015). The body is not a simple object: it is perceived through a complex multisensory processing, which integrated information from the outside (exteroception) and from the inside (interoception), with prior knowledge about the human body and subjective memories. These different stimuli also interact in the simulative code used by the brain for creating new concepts. Hence, as concepts are embodied simulations, and BIs are embodied solutions, it is possible to facilitate cognitive modelling and change by implementing targeted BIs interventions (Riva et al., 2019). Some of the reviewed clinical studies used BIs toward body parts, such as the RHI to modulate obsessive-compulsive disorders (Jalal et al., 2020), or to modify traumatic memories through embodiment in patients presenting posttraumatic stress disorder (Rabellino et al., 2018). However, these can result insufficient to facilitate brain cognitive modelling. At that point, the introduction of VR systems to design full body embodied interventions has had a high impact. In this review, we found that VR systems allow the creation of virtual FBOIS through which the offenders can be represented into the victims' bodies to improve their empathy levels (Seinfeld et al., 2018). Moreover, patients with depression can be represented in a child or in an adult virtual body to increase self-compassion (Falconer et al., 2016). In line with these studies, a recent review study highlights the potential of VR embodiment in promoting psychological well-being (Aymerich-Franch, 2020). However, to date, few studies used embodiment through body illusions to modulate pathological behaviours, attitudes, or cognition. Indeed, only five studies among the seventy-seven studies directed to clinical populations were directed to improve psychological or cognitive responses. 


\section{BI to modulate body representation}

Most of the studies directed to clinical populations in this systematic review have been directed to modulate altered body representation after suffering a pathological condition such as in chronic pain, eating disorders, or brain damage. In this regard, the predictive coding hypothesis argue that the brain maintains an internal model of the body and the space around it, i.e. the body matrix, allowing to create predictions about the upcoming sensory stimuli arriving to the body and to optimally interact with the dynamic environment (Barrett, 2017; Riva et al., 2019). Top-down and bottom-up multisensory processes converge into the body matrix and define our self-placed inside the body, the body that our brain has considered to be our body (Apps \& Tsakiris, 2014; Holmes \& Spence, 2006; S. Serino et al., 2018). Hence, through BIs it is possible to experimentally manipulate the multisensory information arriving at the body to modulate selfconsciousness and the body representation in the brain. In this systematic review, we presented a comprehensive overview of works in which BIs have been used to restore altered body representation in patients with psychiatric disorders such as schizophrenia, in which it has been found a perturbed sense of body ownership, and a weak sense of self (Ferri et al., 2014; Lev-Ari et al., 2015; Peled et al., 2003; Prikken et al., 2019; K.N. Thakkar et al., 2011; Van Haren et al., 2018), as well as an altered self-recognition (Sandsten et al., 2020), and the way in patients with schizophrenia discriminate others (Ferroni et al., 2019). Moreover, BIs have been also used in patients with eating disorders (Carey and Preston, 2019; Eshkevari et al., 2012, 2014; Keizer et al., 2014; Tagini et al., 2020; Zopf et al., 2016; Forghieri et al., 2016; Keizer et al., 2016), in which inner body perception is often disturbed presenting distortions or mismatches in discriminating how the body is perceived and how physically it is (Malighetti et al., 2020). In this regard, BIs have been used to show alterations in body perception in patients with EDs compared to a healthy controls (Carey \& Preston, 2019; E. Eshkevari et al., 2012; Ertimiss Eshkevari et al., 2014; Keizer et al., 2014; Tagini et al., 2020; Zopf et al., 2016), as well as to modulate body size estimation of real body parts that are more emotionally salient (e.g. shoulders, abdomen, hips) in patients with AN (Keizer et al., 2016).

It is known that amputee patients suffer a reorganization of the representation of the affected limb in the brain after the amputation. Indeed some amputee patients reported phantom limb distortions, such as the telescoped effect (that is the feeling of the distal part of the phantom limb is perceived as shrinking within the stump) (Schmalzl \& Ehrsson, 2011). In addition, nervous system injuries such as stroke or spinal cord injury can also change the internal bodily representation (Berlucchi \& Aglioti, 2010; Leemhuis et al., 2019). In this systematic review, we found several studies directed at restoring such body representation distortions through the use of BIs toward body parts in patients with neurological disorders and amputee patients (Bolognini et 
al., 2014; Demartini et al., 2016; Fiorio et al., 2014; Fossataro et al., 2018; Garbarini et al., 2015; Jenkinson et al., 2013; Lenggenhager et al., 2012, 2015; Martinaud et al., 2017; Nava et al., 2014; Ng and Singh, 2015; Rae et al., 2018; D’Alonzo et al., 2015; Giummarra et al., 2010b; Imaizumi et al., 2017).

\section{Types of BI and effectiveness of BIs for clinical applications}

Even though the introduction of new technologies such as virtual reality systems have paved the way to the use of FBIs by providing the sense of being embodied in a full virtual body, in this systematic review we found that still few studies are using FBIs to deal with body distortions in clinical populations with mental illness. Indeed, figure 2 shows that only the 9,4\% of the 77 studies using BIs in clinical populations used FBIs. In contrast, $88,2 \%$ of the studies used BIs toward a body part to modify the altered body part representation in clinical populations.

By using virtual FBIs, in addition to generate the sense of embodiment toward a full virtual body, it is possible to modify the embodiment experience by designing targeted virtual environments (Oliveira et al., 2016; Slater \& Sanchez-Vives, 2016). In fact, even though the use of BIs toward body parts have been shown useful to modify body representations, these are not able to generate the feeling of being present and fully embodied in the virtual body and in the surrounded space (Riva et al., 2019).

Nevertheless, even though VR has been shown to be effective as a clinical tool, this still presents some limitations. VR effectively simulates the external world and changes the external characteristics of the virtual body and the virtual environment. Still, VR presents some limitations as it cannot directly manipulate the inner world of participants, including their interoceptive, proprioceptive, and vestibular sensations. In this regard, Riva and colleagues (2017) presented a new concept known as 'sonoception' through which is possible to modify not only the external representation of the body, but also the inner body perception (Riva et al., 2017). The sonoception is a new noninvasive technological paradigm based on wearable acoustic and vibrotactile transducers, as a new approach to modulate, augment, and replace the contents of the inner body (Riva et al., 2017). The first aim of this approach is the development of an interoceptive simulator that can assess interoceptive time perception in clinical populations, as well as enhance heart rate variability (short-term vagally mediated component—rMSSD) (Di Lernia, Serino, et al., 2018), through the modulation of the parasympathetic system (Di Lernia, Cipresso, et al., 2018). Hence, the combination of this new technological approach together with FBIs through virtual bodies will represent an optimal solution to fully modulate internal and external aspects of body 
representation in clinical populations with altered body perception because of the clinical condition.

\section{Conclusions}

This review provide evidence for the promising role of BIs to improve mental health in clinical populations. To date, most of the used BIs for clinical practices are directed to a body part, but few studies used FBIs. Most of the studies using FBIs in clinical populations used virtual reality systems to induce the sense of embodiment toward a full virtual body. Nevertheless, this type of intervention presents some limitations in fully modifying the patients' internal body representation. The new concept of 'sonoception' and virtual FBIs may represent a complete solution to modulate both the internal and the external aspects of body representation, paving the way for a new 'embodied medicine' technology.

\section{References}

Abdulkarim, Z., \& Ehrsson, H. H. (2016). No causal link between changes in hand position sense and feeling of limb ownership in the rubber hand illusion. Attention, Perception, and Psychophysics, 78(2), 707-720. https://doi.org/10.3758/s13414-015-1016-0

Alberto Gallace and Charles Spence. (2014). In Touch with the Future: The Sense of Touch from Cognitive Neuroscience to Virtual Reality. Oxford University Press. https://doi.org/10.1162/pres_r_00182

Alimardani, M., Nishio, S., \& Ishiguro, H. (2013). Humanlike robot hands controlled by brain activity arouse illusion of ownership in operators. Scientific Reports, 3, 2396. https://doi.org/10.1038/srep02396

Alphonso, A. L., Monson, B. T., Zeher, M. J., Armiger, R. S., Weeks, S. R., Burck, J. M., Moran, C., Davoodie, R., Loeb, G., Pasquina, P. F., \& Tsao, J. W. (2012). Use of a virtual integrated environment in prosthetic limb development and phantom limb pain. Annual Review of CyberTherapy and Telemedicine, 10, 305-309.

Anaforoğlu Külünkoğlu, B., Erbahçeci, F., \& Alkan, A. (2019). A comparison of the effects of mirror therapy and phantom exercises on phantom limb pain. Turkish Journal of Medical Sciences, 49(1), 101-109. https://doi.org/10.3906/sag-1712-166

Apps, M. A. J., \& Tsakiris, M. (2014). The free-energy self: A predictive coding account of self-recognition. Neuroscience \& Biobehavioral Reviews, 41, 85-97. 
https://doi.org/10.1016/j.neubiorev.2013.01.029

Aspell, J. E., Lenggenhager, B., \& Blanke, O. (2009). Keeping in touch with one's self: multisensory mechanisms of self-consciousness. PloS One, 4(8), e6488. https://doi.org/10.1371/journal.pone.0006488

Aymerich-Franch, L. (2020). Avatar embodiment experiences to enhance mental health. In Technology and Health (pp. 49-66). https://doi.org/10.1016/b978-0-12-816958-2.00003-4

Banakou, D., Groten, R., \& Slater, M. (2013). Illusory ownership of a virtual child body causes overestimation of object sizes and implicit attitude changes. Proceedings of the National Academy of Sciences of the United States of America, 110(31), 12846-12851. https://doi.org/10.1073/pnas.1306779110

Banakou, D., Hanumanthu, P. D., \& Slater, M. (2016). Virtual Embodiment of White People in a Black Virtual Body Leads to a Sustained Reduction in Their Implicit Racial Bias. Frontiers in Human Neuroscience, 10. https://doi.org/10.3389/fnhum.2016.00601

Banakou, D., Kishore, S., \& Slater, M. (2018). Virtually Being Einstein Results in an Improvement in Cognitive Task Performance and a Decrease in Age. Frontiers in Psychology, 9(June), Article 917. https://doi.org/10.3389/fpsyg.2018.00917

Banakou, D., \& Slater, M. (2014). Body ownership causes illusory self-attribution of speaking and influences subsequent real speaking. 111(49). https://doi.org/10.1073/pnas.1414936111

Barnsley, N., McAuley, J. H., Mohan, R., Dey, a., Thomas, P., \& Moseley, G. L. (2011). The rubber hand illusion increases histamine reactivity in the real arm. Current Biology, 21(23), R945-R946. https://doi.org/10.1016/j.cub.2011.10.039

Barrett, L. F. (2017). The theory of constructed emotion: an active inference account of interoception and categorization. Social Cognitive and Affective Neuroscience, 12(1), 123. https://doi.org/10.1093/scan/nsw154

Bashford, L., \& Mehring, C. (2016). Ownership and agency of an independent supernumerary hand induced by an imitation brain-computer interface. PLOS ONE, 11(6). https://doi.org/10.1371/journal.pone.0156591

Bekrater-Bodmann, R., Foell, J., Diers, M., \& Flor, H. (2012). The perceptual and neuronal stability of the rubber hand illusion across contexts and over time. Brain Research, 1452, 130-139. https://doi.org/10.1016/j.brainres.2012.03.001

Bergouignan, L., Nyberg, L., \& Ehrsson, H. H. (2014). Out-of-body-induced hippocampal 
amnesia. Proceedings of the National Academy of Sciences of the United States of America, 111(12), 4421-4426. https://doi.org/10.1073/pnas.1318801111

Bergström, I., Kilteni, K., \& Slater, M. (2016). First-Person Perspective Virtual Body Posture Influences Stress: A Virtual Reality Body Ownership Study. Plos One, 11(2), e0148060. https://doi.org/10.1371/journal.pone.0148060

Berlucchi, G., \& Aglioti, S. M. (2010). The body in the brain revisited. Experimental Brain Research. Experimentelle Hirnforschung. Expérimentation Cérébrale, 200(1), 25-35. https://doi.org/10.1007/s00221-009-1970-7

Bisiach, E., Rusconi, M. L., \& Vallar, G. (1991). Remission of somatoparaphrenic delusion through vestibular stimulation. Neuropsycholo, 29(10), 1029-1031.

Blackmore, S. (2017). Seeing myself: The new science of out-of-body experiences (Robinson).

Blanke, O. (2012). Multisensory brain mechanisms of bodily self-consciousness. Nature Reviews. Neuroscience, 13(8), 556-571. https://doi.org/10.1038/nrn3292

Blanke, O., Metzinger, T., \& Lenggenhager, B. (2008). How does the brain localize the self? Science, 317(5841), 1239. https://doi.org/10.5167/uzh-139677

Blanke, O., \& Mohr, C. (2005). Out-of-body experience, heautoscopy, and autoscopic hallucination of neurological origin Implications for neurocognitive mechanisms of corporeal awareness and self-consciousness. Brain Research. Brain Research Reviews, 50(1), 184-199. https://doi.org/10.1016/j.brainresrev.2005.05.008

Bogdanov, S., Smith, J., \& Frey, S. H. (2012). Former hand territory activity increases after amputation during intact hand movements, but is unaffected by illusory visual feedback. Neurorehabilitation and Neural Repair, 26(6), 604-615. https://doi.org/10.1177/1545968311429687

Bolognini, N., Ronchi, R., Casati, C., Fortis, P., \& Vallar, G. (2014). Multisensory remission of somatoparaphrenic delusion: My hand is back! Neurology: Clinical Practice, 4, 216-225. https://doi.org/10.1212/CPJ.0000000000000033

Bolognini, N., Russo, C., \& Vallar, G. (2015). Crossmodal illusions in neurorehabilitation. Frontiers in Behavioral Neuroscience, 9(AUGUST). https://doi.org/10.3389/fnbeh.2015.00212

Botvinick, M., \& Cohen, J. (1998). Rubber hands "feel" touch that eyes see [8]. Nature, 391(6669), 756. https://doi.org/10.1038/35784

Botvinick, Matthew. (2004). Probing the Neural Basis of Body Ownership. Science (New York, 
N.Y.), 305, 782-783.

Botvinick, Matthew, \& Cohen, J. (1998). Rubber hands 'feel' touch that eyes see. Nature, 391(6669), 756-756. https://doi.org/10.1038/35784

Braithwaite, J. J., Watson, D. G., \& Dewe, H. (2017). Predisposition to out-of-body experience $(\mathrm{OBE})$ is associated with aberrations in multisensory integration: Psychophysiological support from a "rubber hand illusion" study. Journal of Experimental Psychology: Human Perception and Performance, 43(6), 1125-1143. https://doi.org/10.1037/xhp0000406

Brown, H., \& Friston, K. J. (2012). Free-energy and illusions: the cornsweet effect. Frontiers in Psychology, 3(February), 43. https://doi.org/10.3389/fpsyg.2012.00043

Bufalari, I., Lenggenhager, B., Porciello, G., Holmes, B. S., \& Aglioti, S. M. (2014). Enfacing others but only if they are nice to you. Frontiers in Behavioral Neuroscience, 8(MAR). https://doi.org/10.3389/fnbeh.2014.00102

Burin, D., Kilteni, K., Rabuffetti, M., Slater, M., \& Pia, L. (2019). Body ownership increases the interference between observed and executed movements. PLOS ONE, 14(1). https://doi.org/10.1371/journal.pone.0209899

Capelari, E. D. P., Uribe, C., \& Brasil-Neto, J. P. (2009). Feeling pain in the rubber hand: Integration of visual, proprioceptive, and painful stimuli. Perception, 38(1), 92-99. https://doi.org/10.1068/p5892

Carey, M., \& Preston, C. (2019). Investigating the Components of Body Image Disturbance Within Eating Disorders. Frontiers in Psychiatry, 10. https://doi.org/10.3389/fpsyt.2019.00635

Cha, H. G., \& Oh, D. W. (2016). Effects of mirror therapy integrated with task-Oriented exercise on the balance function of patients with poststroke hemiparesis: A randomizedControlled pilot trial. International Journal of Rehabilitation Research, 39(1), 70-76. https://doi.org/10.1097/MRR.0000000000000148

Chan, A. W. Y., Bilger, E., Griffin, S., Elkis, V., Weeks, S., Hussey-Anderson, L., Pasquina, P. F., Tsao, J. W., \& Baker, C. I. (2019). Visual responsiveness in sensorimotor cortex is increased following amputation and reduced after mirror therapy. NeuroImage: Clinical, 23. https://doi.org/10.1016/j.nicl.2019.101882

Choi, H. S., Shin, W. S., \& Bang, D. H. (2019). Mirror therapy using gesture recognition for upper limb function, neck discomfort, and quality of life after chronic stroke: A singleblind randomized controlled trial. Medical Science Monitor, 25, 3271-3278. https://doi.org/10.12659/MSM.914095 
Christoffersen, G., Petrini, L., \& Arendt-Nielsen, L. (2012). Modulating pain with augmented reality. Scandinavian Journal of Pain, 3(3), 192-192.

https://doi.org/10.1016/j.sjpain.2012.05.051

Coleshill, M. J., George, D. N., \& Mazzoni, G. (2017). Placebo Analgesia From a Rubber Hand. Journal of Pain, 18(9), 1067-1077. https://doi.org/10.1016/j.jpain.2017.04.004

Corno, G., Serino, S., Cipresso, P., Baños, R. M., \& Riva, G. (2018). Assessing the Relationship Between Attitudinal and Perceptual Component of Body Image Disturbance Using Virtual Reality. Cyberpsychology, Behavior, and Social Networking, 21(11), 679-686. https://doi.org/10.1089/cyber.2018.0340

Cristina, L. M., Matei, D., Ignat, B., \& Popescu, C. D. (2015). Mirror therapy enhances upper extremity motor recovery in stroke patients. Acta Neurologica Belgica, 115(4), 597-603.

D’Alonzo, M., Clemente, F., \& Cipriani, C. (2015). Vibrotactile stimulation promotes embodiment of an Alien hand in amputees with phantom sensations. IEEE Transactions on Neural Systems and Rehabilitation Engineering, 23(3), 450-457. https://doi.org/10.1109/TNSRE.2014.2337952

Darnall, B. D., \& Li, H. (2012). Home-based self-delivered mirror therapy for phantom pain: A pilot study. Journal of Rehabilitation Medicine, 44(3), 254-260. https://doi.org/10.2340/16501977-0933

David, N., Fiori, F., \& Aglioti, S. M. (2014). Susceptibility to the rubber hand illusion does not tell the whole body-awareness story. Cognitive, Affective \& Behavioral Neuroscience, 14(1), 297-306. https://doi.org/10.3758/s13415-013-0190-6

de Bézenac, C. E., Sluming, V., Alhazmi, F., \& Corcoran, R. (2018). Agency performance modulates resting-state variation in prefrontal brain regions. Neuropsychologia. https://doi.org/10.1016/j.neuropsychologia.2017.12.035

de Vignemont, F., Ehrsson, H. H., \& Haggard, P. (2005). Bodily illusions modulate tactile perception. Current Biology : CB, 15(14), 1286-1290. https://doi.org/10.1016/j.cub.2005.06.067

Demartini, B., Ricciardi, L., Crucianelli, L., Fotopoulou, A., \& Edwards, M. J. (2016). Sense of body ownership in patients affected by functional motor symptoms (conversion disorder). Consciousness and Cognition, 39, 70-76. https://doi.org/10.1016/j.concog.2015.11.005

Dempsey-Jones, H., \& Kritikos, A. (2014). Higher-order cognitive factors affect subjective but not proprioceptive aspects of self-representation in the rubber hand illusion. Consciousness and Cognition, 26(1), 74-89. https://doi.org/10.1016/j.concog.2014.02.005 
Di Lernia, D., Cipresso, P., Pedroli, E., \& Riva, G. (2018). Toward an embodied medicine: A portable device with programmable interoceptive stimulation for heart rate variability enhancement. Sensors (Switzerland), 18(8). https://doi.org/10.3390/s18082469

Di Lernia, D., Serino, S., Pezzulo, G., Pedroli, E., Cipresso, P., \& Riva, G. (2018). Feel the time. Time perception as a function of interoceptive processing. Frontiers in Human Neuroscience, 12. https://doi.org/10.3389/fnhum.2018.00074

Diers, M., Christmann, C., Koeppe, C., Ruf, M., \& Flor, H. (2010). Mirrored, imagined and executed movements differentially activate sensorimotor cortex in amputees with and without phantom limb pain. Pain, 149(2), 296-304. https://doi.org/10.1016/j.pain.2010.02.020

Dohle, C., Kleiser, R., Seitz, R. J., \& Freund, H. J. (2004). Body Scheme Gates Visual Processing. Journal of Neurophysiology, 91(5), 2376-2379. https://doi.org/10.1152/jn.00929.2003

Dohle, C., Püllen, J., Nakaten, A., Küst, J., Rietz, C., \& Karbe, H. (2009). Mirror therapy promotes recovery from severe hemiparesis: A randomized controlled trial. Neurorehabilitation and Neural Repair, 23(3), 209-217. https://doi.org/10.1177/1545968308324786

Dummer, T, Picot-Annand, A., Neal, T., \& Moore, C. (2009). Movement and the rubber hand illusion. Perception.

Dummer, Timothy, Picot-Annand, A., Neal, T., \& Moore, C. (2009). Movement and the rubber hand illusion. Perception, 38(2), 271-280. https://doi.org/10.1068/p5921

Ehrsson, H. H. (2007). The experimental induction of out-of-body experiences. Science, 317(5841), 1048. https://doi.org/10.1126/science.1142175

Ehrsson, H. H., Holmes, N. P., \& Passingham, R. E. (2005). Touching a rubber hand: feeling of body ownership is associated with activity in multisensory brain areas. The Journal of Neuroscience: The Official Journal of the Society for Neuroscience, 25(45), 10564 10573. https://doi.org/10.1523/JNEUROSCI.0800-05.2005

Ehrsson, H. H., Kito, T., Sadato, N., Passingham, R. E., \& Naito, E. (2005). Neural substrate of body size: illusory feeling of shrinking of the waist. PLoS Biology, 3(12), e412. https://doi.org/10.1371/journal.pbio.0030412

Ehrsson, H. H., Spence, C., \& Passingham, R. E. (2004). That's my hand! Activity in premotor cortex reflects feeling of ownership of a limb. Science (New York, N.Y.), 305(5685), 875877. https://doi.org/10.1126/science.1097011 
Elmagarmid, A., Fedorowicz, Z., Hammady, H., Ilyas, I., Khabsa, M., \& Ouzzani, M. (2014). Rayyan: a systematic reviews web app for exploring and filtering searches for eligible studies for Cochrane Reviews. Evidence-Informed Public Health: Opportunities and Challenges. Abstracts of the 22nd Cochrane Colloquium.

Ensink, K., Berthelot, N., Biberdzic, M., \& Normandin, L. (2016). The mirror paradigm: Assessing the embodied self in the context of abuse. Psychoanalytic Psychology, 33(3), 389-405. https://doi.org/10.1037/pap0000018

Eshkevari, E., Rieger, E., Longo, M. R., Haggard, P., \& Treasure, J. (2012). Increased plasticity of the bodily self in eating disorders. Psychological Medicine, 42(4), 819-828. https://doi.org/10.1017/S0033291711002091

Eshkevari, Ertimiss, Rieger, E., Longo, M. R., Haggard, P., \& Treasure, J. (2014). Persistent body image disturbance following recovery from eating disorders. International Journal of Eating Disorders, 47(4), 400-409. https://doi.org/10.1002/eat.22219

Evans, N., \& Blanke, O. (2013). Shared electrophysiology mechanisms of body ownership and motor imagery. NeuroImage, 64, 216-228. https://doi.org/10.1016/j.neuroimage.2012.09.027

Falconer, C. J., Rovira, A., King, J. A., Gilbert, P., Antley, A., Fearon, P., Ralph, N., Slater, M., \& Brewin, C. R. (2016). Embodying self-compassion within virtual reality and its effects on patients with depression. BJPsych Open, 2(1), 74-80. https://doi.org/10.1192/bjpo.bp.115.002147

Falconer, C. J., Slater, M., Rovira, A., King, J. A., Gilbert, P., Antley, A., \& Brewin, C. R. (2014). Embodying compassion: a virtual reality paradigm for overcoming excessive selfcriticism. PloS One, 9(11), e111933. https://doi.org/10.1371/journal.pone.0111933

Fang, W., Zhang, R., Zhao, Y., Wang, L., \& Zhou, Y. Di. (2019). Attenuation of pain perception induced by the rubber hand illusion. Frontiers in Neuroscience, 13, 261. https://doi.org/10.3389/fnins.2019.00261

Farrer, C., \& Frith, C. D. (2002). Experiencing oneself vs another person as being the cause of an action: The neural correlates of the experience of agency. NeuroImage. https://doi.org/10.1006/nimg.2001.1009

Ferrè, E. R., Berlot, E., \& Haggard, P. (2015). Vestibular contributions to a right-hemisphere network for bodily awareness: Combining galvanic vestibular stimulation and the "Rubber Hand Illusion." Neuropsychologia, 69, 140-147. https://doi.org/10.1016/j.neuropsychologia.2015.01.032 
Ferrer-Garcia, M., Porras-Garcia, B., González-Ibañez, C., Gracia-Blanes, M., Vilalta-Abella, F., Pla-Sanjuanelo, J., Riva, G., Dakanalis, A., Achotegui-Loizate, J., Talarn-Caparrós, A., Ribas-Sabate, J., Andreu-Gracia, A., Díaz-Marsa, M., Monràs-Arnau, M., SerranoTroncoso, E., Treasure, J., \& Gutiérrez-Maldonado, J. (2017). Does owning a "fatter" virtual body increase body anxiety in college students? Annual Review of CyberTherapy and Telemedicine, 15, 147-153.

Ferri, F., Costantini, M., Salone, A., Di Iorio, G., Martinotti, G., Chiarelli, A., Merla, A., Di Giannantonio, M., \& Gallese, V. (2014). Upcoming tactile events and body ownership in schizophrenia. Schizophrenia Research, 152(1), 51-57.

https://doi.org/10.1016/j.schres.2013.06.026

Ferroni, F., Ardizzi, M., Sestito, M., Lucarini, V., Daniel, B. D., Paraboschi, F., Tonna, M., Marchesi, C., \& Gallese, V. (2019). Shared multisensory experience affects Others' boundary: The enfacement illusion in schizophrenia. Schizophrenia Research, 206, 225235. https://doi.org/10.1016/j.schres.2018.11.018

Fink, G. R., Marshall, J. C., Halligan, P. W., Frith, C. D., Driver, J., Frackowiak, R. S. J., \& Dolan, R. J. (1999). The neural consequences of conflict between intention and the senses. Brain, 122(3), 497-512. https://doi.org/10.1093/brain/122.3.497

Finn, S. B., Perry, B. N., Clasing, J. E., Walters, L. S., Jarzombek, S. L., Curran, S., Rouhanian, M., Keszler, M. S., Hussey-Andersen, L. K., Weeks, S. R., Pasquina, P. F., \& Tsao, J. W. (2017). A randomized, controlled trial of mirror therapy for upper extremity phantom limb pain in male amputees. Frontiers in Neurology, 8(JUL). https://doi.org/10.3389/fneur.2017.00267

Fiorio, M., Mariotti, C., Panzeri, M., Antonello, E., Classen, J., \& Tinazzi, M. (2014). The role of the cerebellum in dynamic changes of the sense of body ownership: A study in patients with cerebellar degeneration. Journal of Cognitive Neuroscience, 26(4), 712-721. https://doi.org/10.1162/jocn_a_00522

Foell, J., Bekrater-Bodmann, R., Diers, M., \& Flor, H. (2014). Mirror therapy for phantom limb pain: Brain changes and the role of body representation. European Journal of Pain (United Kingdom), 18(5), 729-739. https://doi.org/10.1002/j.1532-2149.2013.00433.x

Folegatti, A., de Vignemont, F., Pavani, F., Rossetti, Y., \& Farnè, A. (2009). Losing one's hand: visual-proprioceptive conflict affects touch perception. PloS One, 4(9), e6920. https://doi.org/10.1371/journal.pone.0006920

Folegatti, A., Farnè, A., Salemme, R., \& de Vignemont, F. (2012). The Rubber Hand Illusion: 
two's a company, but three's a crowd. Consciousness and Cognition, 21(2), 799-812. https://doi.org/10.1016/j.concog.2012.02.008

Forghieri, M., Monzani, D., Mackinnon, A., Ferrari, S., Gherpelli, C., \& Galeazzi, G. M. (2016). Posturographic destabilization in eating disorders in female patients exposed to body image related phobic stimuli. Neuroscience Letters, 629, 155-159. https://doi.org/10.1016/j.neulet.2016.07.002

Fossataro, C., Bruno, V., Gindri, P., Pia, L., Berti, A., \& Garbarini, F. (2018). Feeling touch on the own hand restores the capacity to visually discriminate it from someone else' hand: Pathological embodiment receding in brain-damaged patients. Cortex, 104, 207-219. https://doi.org/10.1016/j.cortex.2017.06.004

Friston, K. J. (2009). The free-energy principle: a rough guide to the brain? Trends in Cognitive Sciences, 13(7), 293-301. https://doi.org/10.1016/j.tics.2009.04.005

Friston, K. J., Daunizeau, J., Kilner, J., \& Kiebel, S. J. (2010). Action and behavior: A freeenergy formulation. Biological Cybernetics, 102(3), 227-260. https://doi.org/10.1007/s00422-010-0364-z

Garbarini, F., Fossataro, C., Berti, A., Gindri, P., Romano, D., Pia, L., della Gatta, F., Maravita, A., \& Neppi-Modona, M. (2015). When your arm becomes mine: Pathological embodiment of alien limbs using tools modulates own body representation. Neuropsychologia, 70, 402-413. https://doi.org/10.1016/j.neuropsychologia.2014.11.008

Garry, M. I., Loftus, A., \& Summers, J. J. (2005). Mirror, mirror on the wall: Viewing a mirror reflection of unilateral hand movements facilitates ipsilateral M1 excitability. Experimental Brain Research, 163(1), 118-122. https://doi.org/10.1007/s00221-005-22269

Germine, L., Benson, T. L., Cohen, F., \& Hooker, C. I. L. (2013). Psychosis-proneness and the rubber hand illusion of body ownership. Psychiatry Research, 207(1-2), 45-52. https://doi.org/10.1016/j.psychres.2012.11.022

Gilmour, H. (2014). Positive mental health and mental illness. Health Reports, 25(9), 3-9.

Giummarra, M. J., Fitzgibbon, B. M., Georgiou-Karistianis, N., Nicholls, M. E. R., Gibson, S. J., \& Bradshaw, J. L. (2010). Ouch! my phantom leg jumps/hurts when you stab "my" virtual hand. Perception, 39(10), 1396-1407. https://doi.org/10.1068/p6582

Giummarra, M. J., Georgiou-Karistianis, N., Nicholls, M. E. R., Gibson, S. J., \& Bradshaw, J. L. (2010). The phantom in the mirror: A modified rubber-hand illusion in amputees and normals. Perception, 39(1), 103-118. https://doi.org/10.1068/p6519 
Giummarra, M. J., Gibson, S. J., Georgiou-Karistianis, N., \& Bradshaw, J. L. (2007). Central mechanisms in phantom limb perception: The past, present and future. Brain Research Reviews, 54(1), 219-232. https://doi.org/10.1016/j.brainresrev.2007.01.009

Gonzalez-Franco, M., Cohn, B., Burin, D., \& Maselli, A. (2020). The Self-Avatar Follower Effect in Virtual Reality. IEEE Conference on Virtual Reality and 3D User Interfaces, 8.

Goodwin, G. M., McCloskey, D. I., \& Matthews, P. B. C. (1972). Proprioceptive illusions induced by muscle vibration: contribution by muscle spindles to perception? Science, 175(4028), 1382-1384.

Graziano, M. S. A., \& Botvinick, M. (2002). How the brain represents the body: insights from neurophysiology and psychology. In W. Prinz and B. Hommel (Ed.), Common Mechanisms in Perception and Action: Attention and Performance (XIX., pp. 136-157). Oxford University Press, Oxford England.

Grunt, S., Newman, C. J., Saxer, S., Steinlin, M., Weisstanner, C., \& Kaelin-Lang, A. (2017). The Mirror Illusion Increases Motor Cortex Excitability in Children with and Without Hemiparesis. Neurorehabilitation and Neural Repair, 31(3), 280-289. https://doi.org/10.1177/1545968316680483

Guterstam, A., \& Ehrsson, H. H. (2012). Disowning one's seen real body during an out-of-body illusion. Consciousness and Cognition, 21(2), 1037-1042. https://doi.org/10.1016/j.concog.2012.01.018

Guterstam, A., Gentile, G., \& Ehrsson, H. H. (2013). The Invisible Hand Illusion : Multisensory Integration Leads to the Embodiment of a Discrete Volume of Empty Space. Journal of Cognitive Neuroscience, 25(7), 1078-1099. https://doi.org/10.1162/jocn

Guterstam, A., Petkova, V. I., \& Ehrsson, H. H. (2011). The illusion of owning a third arm. PloS One, 6(2), e17208. https://doi.org/10.1371/journal.pone.0017208

Hadoush, H., Mano, H., Sunagawa, T., Nakanishi, K., \& Ochi, M. (2013). Optimization of mirror therapy to excite ipsilateral primary motor cortex. NeuroRehabilitation, 32(3), 617624. https://doi.org/10.3233/NRE-130884

Haggard, P., \& Jundi, S. (2009). Rubber hand illusions and size - weight illusions: Selfrepresentation modulates representation of external objects. Perception, 38(12), 17961803. https://doi.org/10.1068/p6399

Hamilton-Giachritsis, C., Banakou, D., Garcia Quiroga, M., Giachritsis, C., \& Slater, M. (2018). Reducing risk and improving maternal perspective-taking and empathy using virtual embodiment. Scientific Reports, 8(1). https://doi.org/10.1038/s41598-018-21036-2 
Hänsel, A., Lenggenhager, B., von Känel, R., Curatolo, M., \& Blanke, O. (2011). Seeing and identifying with a virtual body decreases pain perception. European Journal of Pain (London, England), 15(8), 874-879. https://doi.org/10.1016/j.ejpain.2011.03.013

Heed, T., Gründler, M., Rinkleib, J., Rudzik, F. H., Collins, T., Cooke, E., \& O’Regan, J. K. (2011). Visual information and rubber hand embodiment differentially affect reach-tograsp actions. Acta Psychologica, 138(1), 263-271. https://doi.org/10.1016/j.actpsy.2011.07.003

Hohwy, J., \& Paton, B. (2010). Explaining away the body: experiences of supernaturally caused touch and touch on non-hand objects within the rubber hand illusion. PloS One, 5(2), e9416. https://doi.org/10.1371/journal.pone.0009416

Holmes, N. P., Snijders, H. J., \& Spence, C. (2006). Reaching with alien limbs: Visual exposure to prosthetic hands in a mirror biases proprioception without accompanying illusions of ownership. Perception and Psychophysics, 68(4), 685-701. https://doi.org/10.3758/BF03208768

Holmes, N. P., \& Spence, C. (2006). The body schema and the multisensory representation(s) of peripersonal space. Cognitive Processing, 5(2), 94-105. https://doi.org/10.1007/s10339004-0013-3.The

Hwang, H., Cho, S., \& Lee, J. H. (2014). The effect of virtual body swapping with mental rehearsal on pain intensity and body perception disturbance in complex regional pain syndrome. International Journal of Rehabilitation Research, 37(2), 167-172. https://doi.org/10.1097/MRR.0000000000000053

Imaizumi, S., Asai, T., \& Koyama, S. (2017). Agency over phantom limb enhanced by shortterm mirror therapy. Frontiers in Human Neuroscience, 11. https://doi.org/10.3389/fnhum.2017.00483

In, T., Lee, K., \& Song, C. (2016). Virtual reality reflection therapy improves balance and gait in patients with chronic stroke: Randomized controlled trials. Medical Science Monitor, 22, 4046-4053. https://doi.org/10.12659/MSM.898157

Ionta, S., Martuzzi, R., Salomon, R., \& Blanke, O. (2014). The brain network reflecting bodily self-consciousness: a functional connectivity study. Social Cognitive and Affective Neuroscience, 1-10. https://doi.org/10.1093/scan/nst185

Jackson, P. L., \& Decety, J. (2004). Motor cognition: a new paradigm to study self-other interactions. Current Opinion in Neurobiology, 14(2), 259-263. https://doi.org/10.1016/j.conb.2004.01.020 
Jalal, B., McNally, R. J., Elias, J. A., Potluri, S., \& Ramachandran, V. S. (2020). "Fake it till You Make it"! Contaminating Rubber Hands ("Multisensory Stimulation Therapy") to Treat Obsessive-Compulsive Disorder. Frontiers in Human Neuroscience, 13. https://doi.org/10.3389/fnhum.2019.00414

Jenkinson, P. M., Haggard, P., Ferreira, N. C., \& Fotopoulou, A. (2013). Body ownership and attention in the mirror: Insights from somatoparaphrenia and the rubber hand illusion. Neuropsychologia, 1-10. https://doi.org/10.1016/j.neuropsychologia.2013.03.029

Ji, S. G., \& Kim, M. K. (2014). The effects of mirror therapy on the gait of subacute stroke patients: A randomized controlled trial. Clinical Rehabilitation, 29(4), 348-354. https://doi.org/10.1177/0269215514542356

Johnson, M. I., Smith, E., Yellow, S., \& Mulvey, M. R. (2016). A preliminary investigation into psychophysiological effects of threatening a perceptually embodied rubber hand in healthy human participants. Scandinavian Journal of Pain, 11, 1-8. https://doi.org/10.1016/j.sjpain.2015.10.004

Kalckert, A., \& Ehrsson, H. H. (2012). Moving a Rubber Hand that Feels Like Your Own: A Dissociation of Ownership and Agency. Frontiers in Human Neuroscience, 6(March), 114. https://doi.org/10.3389/fnhum.2012.00040

Kammers, M. P. M., de Vignemont, F., Verhagen, L., \& Dijkerman, H. C. (2009). The rubber hand illusion in action. Neuropsychologia, 47(1), 204-211. https://doi.org/10.1016/j.neuropsychologia.2008.07.028

Kang, J. A., Chun, M. H., Choi, S. J., Chang, M. C., \& Yi, Y. G. (2017). Effects of mirror therapy using a tablet $\mathrm{PC}$ on central facial paresis in stroke patients. Annals of Rehabilitation Medicine, 41(3), 347-353. https://doi.org/10.5535/arm.2017.41.3.347

Kang, Y. J., Ku, J., Kim, H. J., Park, H. K., Tabei, Y., Saito, K., Yagi, K., Hallett, M., Katayama, Y., \& Lucking, C. (2011). Facilitation of Corticospinal Excitability According to Motor Imagery and Mirror Therapy in Healthy Subjects and Stroke Patients. Annals of Rehabilitation Medicine, 35(6), 747. https://doi.org/10.5535/arm.2011.35.6.747

Kazemi, F.Elahin, M.Moradi, \& M.Fesharaki. (2015). Effects of mirror therapy on phantom pain in patients with bilateral amputations admitted to the veterans foundation of Qazvin, Iran. Avicenna Journal of Phytomedicine, 5, 103.

Keizer, A., Smeets, M. A. M., Postma, A., van Elburg, A., \& Dijkerman, H. C. (2014). Does the experience of ownership over a rubber hand change body size perception in anorexia nervosa patients? Neuropsychologia, 62(1), 26-37. 
https://doi.org/10.1016/j.neuropsychologia.2014.07.003

Keizer, A., Van Elburg, A., Helms, R., \& Dijkerman, H. C. (2016). A virtual reality full body illusion improves body image disturbance in anorexia nervosa. PLoS ONE, 11(10), e0163921. https://doi.org/10.1371/journal.pone.0163921

Kilteni, K., Bergstrom, I., \& Slater, M. (2013). Drumming in immersive virtual reality: the body shapes the way we play. IEEE Transactions on Visualization and Computer Graphics, 19(4), 597-605. https://doi.org/10.1109/TVCG.2013.29

Kilteni, K., \& Ehrsson, H. H. (2017). Body ownership determines the attenuation of selfgenerated tactile sensations. Proceedings of the National Academy of Sciences, 114(31), 8426-8431. https://doi.org/10.1073/pnas.1703347114

Kilteni, K., Grau-Sánchez, J., De Las Heras, M. V., Rodríguez-Fornells, A., \& Slater, M. (2016). Decreased corticospinal excitability after the illusion of missing part of the arm. Frontiers in Human Neuroscience, 10(APR2016), 1-12. https://doi.org/10.3389/fnhum.2016.00145

Kilteni, K., Maselli, A., Koerding, K., \& Slater, M. (2015). Over my fake body: body ownership illusions for studying the multisensory basis of own-body perception. Frontiers in Human Neuroscience, 9(141), DOI: 10.3389/fnhum.2015.00141. https://doi.org/10.3389/fnhum.2015.00141

Kilteni, K., Normand, J.-M., Sanchez-Vives, M. V, \& Slater, M. (2012). Extending body space in immersive virtual reality: a very long arm illusion. PloS One, 7(7), e40867. https://doi.org/10.1371/journal.pone.0040867

Kim, J. H., \& Lee, B. H. (2015). Mirror therapy combined with biofeedback functional electrical stimulation for motor recovery of upper extremities after stroke: A pilot randomized controlled trial. Occupational Therapy International, 22(2), 51-60. https://doi.org/10.1002/oti.1384

Kojima, K., Ikuno, K., Morii, Y., Tokuhisa, K., Morimoto, S., \& Shomoto, K. (2014). Feasibility study of a combined treatment of electromyography-triggered neuromuscular stimulation and mirror therapy in stroke patients: A randomized crossover trial. NeuroRehabilitation, 34(2), 235-244. https://doi.org/10.3233/NRE-131038

Kokkinara, E., Kilteni, K., Blom, K. J., \& Slater, M. (2016). First Person Perspective of Seated Participants over a Walking Virtual Body Leads to Illusory Agency over the Walking. Scientific Reports, 6(July 2015), 1-11. https://doi.org/10.1038/srep28879

Kong, G., He, K., \& Wei, K. (2017). Sensorimotor experience in virtual reality enhances sense 
of agency associated with an avatar. Consciousness and Cognition, 52, 115-124.

https://doi.org/10.1016/j.concog.2017.04.018

Kühn, S., Brass, M., \& Haggard, P. (2013). Feeling in control: Neural correlates of experience of agency. Cortex. https://doi.org/10.1016/j.cortex.2012.09.002

Kumar, V., Arya, K. N., Pandian, S., Sethi, T., \& Samal, S. K. (2016). Mirror therapy in management of somatosensory impairment among post stroke hemiparesis. International Journal of Stroke, 11, 133-. https://doi.org/10.1177/1747493016670567

Lackner, J. R. (1988). Some proprioceptive influences on the perceptual representation of body shape and orientation. Brain, 111, 281-297.

Lackner, J. R., \& Levine, M. S. (1978). Visual direction depends on the opertion of spatial constancy mechanisms: the oculobrachial illusion. Neuroscience Letters, 7, 207-212.

Leemhuis, De Gennaro, \& Pazzaglia. (2019). Disconnected Body Representation:

Neuroplasticity Following Spinal Cord Injury. Journal of Clinical Medicine, 8(12), 2144. https://doi.org/10.3390/jcm8122144

Lenggenhager, B. (2007). Video Ergo Sum : Manipulating Bodily Self-Consciousness. Science, 1096(2007), 1095-1099. https://doi.org/10.1126/science.1143439

Lenggenhager, B., Hilti, L., \& Brugger, P. (2015). Disturbed body integrity and the "rubber foot illusion.” Neuropsychology, 29(2), 205-211. https://doi.org/10.1037/neu0000143

Lenggenhager, B., Pazzaglia, M., Scivoletto, G., Molinari, M., \& Aglioti, S. M. (2012). The Sense of the Body in Individuals with Spinal Cord Injury. PLoS ONE, 7(11). https://doi.org/10.1371/journal.pone.0050757

Lenggenhager, B., Tadi, T., Metzinger, T., \& Blanke, O. (2007). SOM: Video ergo sum: manipulating bodily self-consciousness. Science (New York, N.Y.), 317(5841), 1096-1099. https://doi.org/10.1126/science.1143439

Lev-Ari, L., Hirschmann, S., Dyskin, O., Goldman, O., \& Hirschmann, I. (2015). The Rubber Hand Illusion paradigm as a sensory learning process in patients with schizophrenia. European Psychiatry, 30(7), 868-873. https://doi.org/10.1016/j.eurpsy.2015.06.008

Limanowski, J., \& Blankenburg, F. (2016). That's not quite me: Limb ownership encoding in the brain. Social Cognitive and Affective Neuroscience, 11(7), 1130-1140. https://doi.org/10.1093/scan/nsv079

Lin, K. C., Huang, P. C., Chen, Y. T., Wu, C. Y., \& Huang, W. L. (2014). Combining afferent stimulation and mirror therapy for rehabilitating motor function, motor control, 
ambulation, and daily functions after stroke. Neurorehabilitation and Neural Repair, 28(2), 153-162. https://doi.org/10.1177/1545968313508468

Llobera, J., Sanchez-Vives, M. V., \& Slater, M. (2013). The relationship between virtual body ownership and temperature sensitivity. Journal of the Royal Society Interface, 10(85), 20130300. https://doi.org/10.1098/rsif.2013.0300

Llobera, J., \& Slater, M. (2013). The relationship between virtual body ownership and temperature sensitivity. May.

Lloyd, D. M. (2007). Spatial limits on referred touch to an alien limb may reflect boundaries of visuo-tactile peripersonal space surrounding the hand. Brain and Cognition, 64(1), 104109. https://doi.org/10.1016/j.bandc.2006.09.013

Longo, M. R. (2015). Implicit and explicit body representations. European Psychologist, 20(1), 6-15. https://doi.org/10.1027/1016-9040/a000198

Longo, M. R., Cardozo, S., \& Haggard, P. (2008). Visual enhancement of touch and the bodily self. Consciousness and Cognition, 17(4), 1181-1191. https://doi.org/10.1016/j.concog.2008.01.001

Longo, M. R., Kammers, M. P. M., Gomi, H., Tsakiris, M., \& Haggard, P. (2009). Contraction of body representation induced by proprioceptive conflict. Current Biology : CB, 19(17), R727-8. https://doi.org/10.1016/j.cub.2009.07.024

Lopez-Carballo, J., Rodriguez, N., Soler, D., Opisso, E., \& Sbert, M. (2018). Gestural interaction and visual illusion for lower limbs' neuropathic pain treatment. IEEE Transactions on Neural Systems and Rehabilitation Engineering, 26(11), 2217-2225. https://doi.org/10.1109/TNSRE.2018.2873593

Lopez, C., Lenggenhager, B., \& Blanke, O. (2010). How vestibular stimulation interacts with illusory hand ownership. Consciousness and Cognition, 19(1), 33-47. https://doi.org/10.1016/j.concog.2009.12.003

Lotze, M., \& Moseley, G. L. (2007). Role of distorted body image in pain. Current Rheumatology Reports, 9(6), 488-496. https://doi.org/10.1007/s11926-007-0079-x

Louw, A., Puentedura, E. J., Reese, D., Parker, P., Miller, T., \& Mintken, P. E. (2017). Immediate Effects of Mirror Therapy in Patients With Shoulder Pain and Decreased Range of Motion. Archives of Physical Medicine and Rehabilitation, 98(10), 1941-1947. https://doi.org/10.1016/j.apmr.2017.03.031

Louzolo, A., Kalckert, A., \& Petrovic, P. (2015). When passive feels active - Delusion- 
proneness alters self-recognition in the moving rubber hand illusion. PLOS ONE, 10(6). https://doi.org/10.1371/journal.pone.0128549

Macauda, G., Bertolini, G., Palla, A., Straumann, D., Brugger, P., \& Lenggenhager, B. (2015). Binding body and self in visuo-vestibular conflicts. European Journal of Neuroscience, 41(6), 810-817. https://doi.org/10.1111/ejn.12809

Maister, L., Sebanz, N., Knoblich, G., \& Tsakiris, M. (2013). Experiencing ownership over a dark-skinned body reduces implicit racial bias. Cognition, 128(2), 170-178. https://doi.org/10.1016/j.cognition.2013.04.002

Maister, L., Slater, M., Sanchez-Vives, M. V., \& Tsakiris, M. (2015). Changing bodies changes minds: Owning another body affects social cognition. Trends in Cognitive Sciences, 19(1), 6-12. https://doi.org/10.1016/j.tics.2014.11.001

Makin, T. R., Holmes, N. P., \& Ehrsson, H. H. (2008). On the other hand: dummy hands and peripersonal space. Behavioural Brain Research, 191(1), 1-10. https://doi.org/10.1016/j.bbr.2008.02.041

Malighetti, C., Gaudio, S., DI LERNIA, D., Matamala-Gomez, M., \& Riva, G. (2020). Altered inner body perception in anorexia and bulimia nervosa: a systematic review.

Martinaud, O., Besharati, S., Jenkinson, P. M., \& Fotopoulou, A. (2017). Ownership illusions in patients with body delusions: Different neural profiles of visual capture and disownership. Cortex, 87, 174-185. https://doi.org/10.1016/j.cortex.2016.09.025

Martini, M., Perez-Marcos, D., \& Sanchez-Vives, M. V. (2013). What colour is my arm? Changes in skin colour of an embodied virtual arm modulates pain threshold. Frontiers in Human Neuroscience, JUL. https://doi.org/10.3389/fnhum.2013.00438

Martini, Matteo, Kilteni, K., Maselli, A., \& Sanchez-vives, M. V. (2015). The body fades away : investigating the effects of transparency of an embodied virtual body on pain threshold and body ownership. Scientific Reports, 5, 13948. https://doi.org/10.1038/srep13948

Martini, Matteo, Perez-Marcos, D., \& Sanchez-Vives, M. V. (2014). Modulation of pain threshold by virtual body ownership. European Journal of Pain (United Kingdom), 18(7), 1040-1048. https://doi.org/10.1002/j.1532-2149.2014.00451.x

Maselli, A. (2015). Allocentric and egocentric manipulations of the sense of self-location in full-body illusions and their relation with the sense of body ownership. Cognitive Processing, 16(2). https://doi.org/10.1007/s10339-015-0667-z

Maselli, A., Kilteni, K., López-Moliner, J., \& Slater, M. (2016). The sense of body ownership 
relaxes temporal constraints for multisensory integration. Scientific Reports, 6(April), 30628. https://doi.org/10.1038/srep30628

Maselli, A., \& Slater, M. (2013). The building blocks of the full body ownership illusion. Frontiers in Human Neuroscience, 7, 83. https://doi.org/10.3389/fnhum.2013.00083

Maselli, A., \& Slater, M. (2014). Sliding perspectives: dissociating ownership from self-location during full body illusions in virtual reality. Frontiers in Human Neuroscience, 8(September), 693. https://doi.org/10.3389/fnhum.2014.00693

Matamala-gomez, M., Donegan, T., Bottiroli, S., Sandrini, G., Sanchez-vives, M. V, Tassorelli, C., \& Matamala-gomez, M. (2019). Immersive Virtual Reality and Virtual Embodiment for Pain Relief. 13(August), 1-12. https://doi.org/10.3389/fnhum.2019.00279

Matamala-gomez, M., Gonzalez, A. M. D., Slater, M., \& Sanchez-vives, M. V. (2018). Decreasing pain ratings in chronic arm pain through changing a virtual body: different strategies for different pain types. The Journal of Pain. https://doi.org/10.1016/j.jpain.2018.12.001

Matamala-Gomez, M., Nierula, B., Donegan, T., Slater, M., \& Sanchez-Vives, M. V. (2020). Manipulating the Perceived Shape and Color of a Virtual Limb Can Modulate Pain Responses. https://doi.org/10.3390/jcm9020291

Matthys, K., Smits, M., Van der Geest, J. N., Van der Lugt, A., Seurinck, R., Stam, H. J., \& Selles, R. W. (2009). Mirror-Induced Visual Illusion of Hand Movements: A Functional Magnetic Resonance Imaging Study. Archives of Physical Medicine and Rehabilitation, 90(4), 675-681. https://doi.org/10.1016/j.apmr.2008.09.571

Melzack, R. (1992). Phantom Limbs. Scientific American, 266(4), 120-125. https://doi.org/10.1038/scientificamerican0492-120

Michielsen, M. E., Smits, M., Ribbers, G. M., Stam, H. J., Van Der Geest, J. N., Bussmann, J. B. J., \& Selles, R. W. (2011). The neuronal correlates of mirror therapy: An fMRI study on mirror induced visual illusions in patients with stroke. Journal of Neurology, Neurosurgery and Psychiatry, 82(4), 393-398. https://doi.org/10.1136/jnnp.2009.194134

Miles, E., Poliakoff, E., \& Brown, R. J. (2011). Medically unexplained symptom reports are associated with a decreased response to the rubber hand illusion. Journal of Psychosomatic Research, 71(4), 240-244. https://doi.org/10.1016/j.jpsychores.2011.04.002

Miller, L. E., Longo, M. R., \& Saygin, A. P. (2017). Visual illusion of tool use recalibrates tactile perception. Cognition, 162, 32-40. https://doi.org/10.1016/j.cognition.2017.01.022 
Morgan, H. L., Turner, D. C., Corlett, P. R., Absalom, A. R., Adapa, R., Arana, F. S., Pigott, J., Gardner, J., Everitt, J., Haggard, P., \& Fletcher, P. C. (2011). Exploring the impact of ketamine on the experience of illusory body ownership. Biological Psychiatry, 69(1), 3541. https://doi.org/10.1016/j.biopsych.2010.07.032

Moseley, G. L., \& Flor, H. (2012). Targeting cortical representations in the treatment of chronic pain: a review. Neurorehabilitation and Neural Repair, 26(6), 646-652. https://doi.org/10.1177/1545968311433209

Moseley, G. L., Gallace, A., \& Spence, C. (2008). Is mirror therapy all it is cracked up to be? Current evidence and future directions. Pain, 138(1), 7-10. https://doi.org/10.1016/j.pain.2008.06.026

Moseley, G. L., Gallace, A., \& Spence, C. (2012). Bodily illusions in health and disease: physiological and clinical perspectives and the concept of a cortical "body matrix". Neuroscience and Biobehavioral Reviews, 36(1), 34-46. https://doi.org/10.1016/j.neubiorev.2011.03.013

Moseley, G. L., Olthof, N., Venema, A., Don, S., Wijers, M., Gallace, A., \& Spence, C. (2008). Psychologically induced cooling of a specific body part caused by the illusory ownership of an artificial counterpart. Proceedings of the National Academy of Sciences of the United States of America, 105(35), 13169-13173. https://doi.org/10.1073/pnas.0803768105

Mulvey, M. R., Fawkner, H. J., Radford, H. E., \& Johnson, M. I. (2012). Perceptual embodiment of prosthetic limbs by transcutaneous electrical nerve stimulation. Neuromodulation, 15(1), 42-47. https://doi.org/10.1111/j.1525-1403.2011.00408.x

Mussap, A. J., \& Salton, N. (2006). A "rubber-hand" illusion reveals a relationship between perceptual body image and unhealthy body change. Journal of Health Psychology, 11(4), 627-639. https://doi.org/10.1177/1359105306065022

Nava, E., Steiger, T., \& Röder, B. (2014). Both developmental and adult vision shape body representations. Scientific Reports, 4, 6622. https://doi.org/10.1038/srep06622

Newport, R., Pearce, R., \& Preston, C. (2010). Fake hands in action: embodiment and control of supernumerary limbs. Experimental Brain Research, 204(3), 385-395. https://doi.org/10.1007/s00221-009-2104-y

Ng, M. J., \& Singh, P. (2015). Mirror therapy in unilateral neglect after stroke (must trial): A randomized controlled trial. Neurology, 84(12), 1286. https://doi.org/10.1212/01.wnl.0000462978.57606.5a

Nierula, B., Martini, M., Matamala-Gomez, M., Slater, M., \& Sanchez-Vives, M. V. (2017). 
Seeing an Embodied Virtual Hand is Analgesic Contingent on Colocation. Journal of Pain, 18(6), 645-655. https://doi.org/10.1016/j.jpain.2017.01.003

Normand, J.-M., Giannopoulos, E., Spanlang, B., \& Slater, M. (2011). Multisensory stimulation can induce an illusion of larger belly size in immersive virtual reality. PloS One, 6(1), e16128. https://doi.org/10.1371/journal.pone.0016128

Ocklenburg, S., Peterburs, J., Rüther, N., \& Güntürkün, O. (2012). The rubber hand illusion modulates pseudoneglect. Neuroscience Letters, 523(2), 158-161. https://doi.org/10.1016/j.neulet.2012.06.068

Ocklenburg, S., Rüther, N., Peterburs, J., Pinnow, M., \& Güntürkün, O. (2011). Laterality in the rubber hand illusion. Laterality, 16(2), 174-187. https://doi.org/10.1080/13576500903483515

Ol, H. S., Van Heng, Y., Danielsson, L., \& Husum, H. (2018). Mirror therapy for phantom limb and stump pain: A randomized controlled clinical trial in landmine amputees in Cambodia. Scandinavian Journal of Pain. https://doi.org/10.1515/sjpain-2018-0042

Oliveira, E., Bertrand, P., Lesur, M., Palomo, P., Demarzo, M., Cebolla, A., Baños, R., \& Tori, R. (2016). Virtual Body Swap: A New Feasible Tool to Be Explored in Health and Education.

Ortiz-Catalan, M., Guðmundsdóttir, R. A., Kristoffersen, M. B., Zepeda-Echavarria, A., CaineWinterberger, K., Kulbacka-Ortiz, K., Widehammar, C., Eriksson, K., Stockselius, A., Ragnö, C., Pihlar, Z., Burger, H., \& Hermansson, L. (2016). Phantom motor execution facilitated by machine learning and augmented reality as treatment for phantom limb pain: a single group, clinical trial in patients with chronic intractable phantom limb pain. The Lancet, 388(10062), 2885-2894. https://doi.org/10.1016/S0140-6736(16)31598-7

Osimo, S. A., Pizarro, R., Spanlang, B., \& Slater, M. (2015). Conversations between self and self as Sigmund Freud-A virtual body ownership paradigm for self counselling. Scientific Reports, 5(July), 13899. https://doi.org/10.1038/srep13899

Osumi, M., Inomata, K., Inoue, Y., Otake, Y., Morioka, S., \& Sumitani, M. (2019). Characteristics of phantom limb pain alleviated with virtual reality rehabilitation. Pain Medicine (United States), 20(5), 1038-1046. https://doi.org/10.1093/pm/pny269

Palmer, C. J., Paton, B., Hohwy, J., \& Enticott, P. G. (2013). Movement under uncertainty: The effects of the rubber-hand illusion vary along the nonclinical autism spectrum. Neuropsychologia, 51(10), 1942-1951. https://doi.org/10.1016/j.neuropsychologia.2013.06.020 
Palmer, C. J., Paton, B., Kirkovski, M., Enticott, P. G., \& Hohwy, J. (2015). Context sensitivity in action decreases along the autism spectrum: A predictive processing perspective. Proceedings of the Royal Society B: Biological Sciences, 282(1802), 1-9. https://doi.org/10.1098/rspb.2014.1557

Palomo, P., Borrego, A., Cebolla, A., Llorens, R., Demarzo, M., \& Baños, R. M. (2018). Subjective, behavioral, and physiological responses to the rubber hand illusion do not vary with age in the adult phase. Consciousness and Cognition, 58, 90-96. https://doi.org/10.1016/j.concog.2017.10.014

Pamment, J., \& Aspell, J. E. (2017). Putting pain out of mind with an 'out of body' illusion. European Journal of Pain (United Kingdom), 21(2), 334-342. https://doi.org/10.1002/ejp.927

Paolucci, T., Cardarola, A., Colonnelli, P., Ferracuti, G., Gonnella, R., Murgia, M., Santilli, V., Paoloni, M., Bernetti, A., Agostini, F., \& Mangone, M. (2020). Give me a kiss!: An integrative rehabilitative training program with motor imagery and mirror therapy for recovery of facial palsy. European Journal of Physical and Rehabilitation Medicine, 56(1), 58-67. https://doi.org/10.23736/S1973-9087.19.05757-5

Pavone, E. F., Tieri, G., Rizza, G., Tidoni, E., Grisoni, L., \& Aglioti, S. M. (2016). Embodying others in immersive virtual reality: Electro-cortical signatures of monitoring the errors in the actions of an avatar seen from a first-person perspective. Journal of Neuroscience, 36(2), 268-279. https://doi.org/10.1523/JNEUROSCI.0494-15.2016

Peled, A., Pressman, A., Geva, A. B., \& Modai, I. (2003). Somatosensory evoked potentials during a rubber-hand illusion in schizophrenia. Schizophrenia Research, 64(2-3), 157163. https://doi.org/10.1016/S0920-9964(03)00057-4

Perepelkina, O., Romanov, D., Arina, G., Volel, B., \& Nikolaeva, V. (2019). Multisensory mechanisms of body perception in somatoform disorders. Journal of Psychosomatic Research, 127. https://doi.org/10.1016/j.jpsychores.2019.109837

Perez-Marcos, D., Martini, M., Fuentes, C. T., Bellido Rivas, A. I., Haggard, P., \& SanchezVives, M. V. (2018). Selective distortion of body image by asynchronous visuotactile stimulation. Body Image, 24, 55-61. https://doi.org/10.1016/j.bodyim.2017.11.002

Perez-Marcos, D., Slater, M., \& Sanchez-Vives, M. V. (2009). Inducing a virtual hand ownership illusion through a brain-computer interface. NeuroReport, 20(6), 589-594. https://doi.org/10.1097/WNR.0b013e32832a0a2a

Petkova, V., \& Ehrsson, H. (2008). If I were you: perceptual illusion of body swapping. PloS 
One.

Ponzo, S., Kirsch, L. P., Fotopoulou, A., \& Jenkinson, P. M. (2018). Balancing body ownership: Visual capture of proprioception and affectivity during vestibular stimulation.

Neuropsychologia, 117, 311-321. https://doi.org/10.1016/j.neuropsychologia.2018.06.020

Porciello, G., Bufalari, I., Minio-Paluello, I., Di Pace, E., \& Aglioti, S. M. (2018). The 'Enfacement' illusion: A window on the plasticity of the self. In Cortex (Vol. 104, pp. 261-275). https://doi.org/10.1016/j.cortex.2018.01.007

Preston, C., \& Ehrsson, H. H. (2014). Illusory changes in body size modulate body satisfaction in a way that is related to non-clinical eating disorder psychopathology. PLoS ONE, 9(1). https://doi.org/10.1371/journal.pone.0085773

Preston, C., \& Ehrsson, H. H. (2016). Illusory obesity triggers body dissatisfaction responses in the insula and anterior cingulate cortex. Cerebral Cortex, 26(12), 4450-4460. https://doi.org/10.1093/cercor/bhw313

Preston, C., \& Henrik Ehrsson, H. (2018). Implicit and explicit changes in body satisfaction evoked by body size illusions: Implications for eating disorder vulnerability in women. PLoS ONE, 13(6). https://doi.org/10.1371/journal.pone.0199426

Prikken, M., Van der Weiden, A., Baalbergen, H., Hillegers, M. H. J., Kahn, R. S., Aarts, H., \& Van Haren, N. E. M. (2019). Multisensory integration underlying body-ownership experiences in schizophrenia and offspring of patients: A study using the rubber hand illusion paradigm. Journal of Psychiatry and Neuroscience, 44(3), 177-184. https://doi.org/10.1503/jpn.180049

Pyasik, M., Salatino, A., \& Pia, L. (2019). Do movements contribute to sense of body ownership? Rubber hand illusion in expert pianists. Psychological Research, 83(1), 185195. https://doi.org/10.1007/s00426-018-1137-x

Rabellino, D., Burin, D., Harricharan, S., Lloyd, C., Frewen, P. A., McKinnon, M. C., \& Lanius, R. A. (2018). Altered sense of body ownership and agency in posttraumatic stress disorder and its dissociative subtype: A rubber hand illusion study. Frontiers in Human Neuroscience, 12. https://doi.org/10.3389/fnhum.2018.00163

Rae, C. L., Larsson, D. E. O., Eccles, J. A., Ward, J., \& Critchley, H. D. (2018). Subjective embodiment during the rubber hand illusion predicts severity of premonitory sensations and tics in Tourette Syndrome. Consciousness and Cognition, 65, 368-377. https://doi.org/10.1016/j.concog.2018.09.011

Ramachandran, V. S., \& Altschuler, E. L. (2009). The use of visual feedback, in particular 
mirror visual feedback, in restoring brain function. Brain, 132(7), 1693-1710.

https://doi.org/10.1093/brain/awp135

Ramachandran, V. S., Altschuler, E. L., Aglioti, S., Bonazzi, A., Cortese, F., Aglioti, S.,

Smania, N., Atzei, A., Berlucchi, G., Altschuler, E., Hu, J., Altschuler, E., Ramachandran, V., Altschuler, E., Wisdom, S., Stone, L., Foster, C., Galasko, D., Llewellyn, D., ...

Köseoğlu, F. (2009). The use of visual feedback, in particular mirror visual feedback, in restoring brain function. Brain : A Journal of Neurology, 132(Pt 7), 1693-1710. https://doi.org/10.1093/brain/awp135

Ramachandran, V. S., \& Hirstein, W. (1998). The perception of phantom limbs. The D. O. Hebb lecture. Brain, 121(9), 1603-1630. https://doi.org/10.1093/brain/121.9.1603

Ramachandran, V. S., \& Rodgers-Ramachandran, D. (1996). Synaesthesia in phantom limbs induced with mirrors. Proceedings of the Royal Society B: Biological Sciences. https://doi.org/10.1098/rspb.1996.0058

Ramachandran, V. S., Rogers-Ramachandran, D., \& Cobb, S. (1995). Touching the phantom limb. Nature, 377(6549), 489-490. https://doi.org/10.1038/377489a0

Ramadugu, S., Nagabushnam, S. C., Katuwal, N., \& Chatterjee, K. (2017). Intervention for phantom limb pain: A randomized single crossover study of mirror therapy. Indian Journal of Psychiatry, 59(4), 457-464. https://doi.org/10.4103/psychiatry.IndianJPsychiatry_259_16

Rao, R. P., \& Ballard, D. H. (1999). Predictive coding in the visual cortex: a functional interpretation of some extra-classical receptive-field effects. Nature Neuroscience, 2(1), 79-87. https://doi.org/10.1038/4580

Reinersmann, A., Landwehrt, J., Krumova, E. K., Peterburs, J., Ocklenburg, S., Güntürkün, O., \& Maier, C. (2013). The rubber hand illusion in complex regional pain syndrome: preserved ability to integrate a rubber hand indicates intact multisensory integration. Pain, 154(9), 1519-1527. https://doi.org/10.1016/j.pain.2013.03.039

Riemer, M., Fuchs, X., Bublatzky, F., Kleinböhl, D., Hölzl, R., \& Trojan, J. (2014). The rubber hand illusion depends on a congruent mapping between real and artificial fingers. Acta Psychologica, 152, 34-41. https://doi.org/10.1016/j.actpsy.2014.07.012

Riemer, M., Wolbers, T., \& Kuehn, E. (2019). Preserved multisensory body representations in advanced age. Scientific Reports, 9(1), 2663. https://doi.org/10.1038/s41598-019-39270-7

Riva, G., \& Dakanalis, A. (2018). Altered processing and integration of multisensory bodily representations and signals in eating disorders: A possible path toward the understanding 
of their underlying causes. Frontiers in Human Neuroscience, 12.

https://doi.org/10.3389/fnhum.2018.00049

Riva, G., Serino, S., Di Lernia, D., Pavone, E. F., \& Dakanalis, A. (2017). Embodied Medicine: Mens Sana in Corpore Virtuale Sano. Frontiers in Human Neuroscience, 11(March), 1-9. https://doi.org/10.3389/fnhum.2017.00120

Riva, G., Wiederhold, B. K., \& Mantovani, F. (2019). Neuroscience of Virtual Reality: From Virtual Exposure to Embodied Medicine. Cyberpsychology, Behavior, and Social Networking, 22(1), 82-96. https://doi.org/10.1089/cyber.2017.29099.gri

Rjosk, V., Kaminski, E., Hoff, M., Sehm, B., Steele, C. J., Villringer, A., \& Ragert, P. (2016). Mirror visual feedback-induced performance improvement and the influence of hand dominance. Frontiers in Human Neuroscience, 9(JAN2016), 702. https://doi.org/10.3389/fnhum.2015.00702

Rjosk, V., Lepsien, J., Kaminski, E., Hoff, M., Sehm, B., Steele, C. J., Villringer, A., \& Ragert, P. (2017). Neural correlates of mirror visual feedback-induced performance improvements: A resting-state fMRI study. Frontiers in Human Neuroscience, 11. https://doi.org/10.3389/fnhum.2017.00054

Romano, D., Bottini, G., \& Maravita, A. (2013). Perceptual effects of the mirror box training in normal subjects. Restorative Neurology and Neuroscience, 31(4), 373-386. https://doi.org/10.3233/RNN-120273

Rothgangel, A., Braun, S., Winkens, B., Beurskens, A., \& Smeets, R. (2018). Traditional and augmented reality mirror therapy for patients with chronic phantom limb pain (PACT study): results of a three-group, multicentre single-blind randomized controlled trial. Clinical Rehabilitation, 32(12), 1591-1608. https://doi.org/10.1177/0269215518785948

Salomon, R., Lim, M., Pfeiffer, C., Gassert, R., \& Blanke, O. (2013). Full body illusion is associated with widespread skin temperature reduction. Frontiers in Behavioral Neuroscience, MAY. https://doi.org/10.3389/fnbeh.2013.00065

Samad, M., Chung, A. J., \& Shams, L. (2015). Perception of Body Ownership Is Driven by Bayesian Sensory Inference. Plos One, 10(2), e0117178. https://doi.org/10.1371/journal.pone.0117178

Samad, M., \& Shams, L. (2018). Recalibrating the body: visuotactile ventriloquism aftereffect. 1-10. https://doi.org/10.7717/peerj.4504

Sanchez-Vives, M. V, Spanlang, B., Frisoli, A., Bergamasco, M., \& Slater, M. (2010). Virtual hand illusion induced by visuomotor correlations. PloS One, 5(4), e10381. 
https://doi.org/10.1371/journal.pone.0010381

Sandsten, K. E., Nordgaard, J., Kjaer, T. W., Gallese, V., Ardizzi, M., Ferroni, F., Petersen, J., \& Parnas, J. (2020). Altered self-recognition in patients with schizophrenia. Schizophrenia Research. https://doi.org/10.1016/j.schres.2020.01.022

Schaefer, M., Flor, H., Heinze, H.-J., \& Rotte, M. (2007). Morphing the body: illusory feeling of an elongated arm affects somatosensory homunculus. NeuroImage, 36(3), 700-705. https://doi.org/10.1016/j.neuroimage.2007.03.046

Schmalzl, L., \& Ehrsson, H. H. (2011). Experimental Induction of a Perceived "Telescoped" Limb Using a Full-Body Illusion. Frontiers in Human Neuroscience. https://doi.org/10.3389/fnhum.2011.00034

Schomaker, J., Tesch, J., Bülthoff, H. H., \& Bresciani, J. P. (2011). It is all me: The effect of viewpoint on visual-vestibular recalibration. Experimental Brain Research, 213(2-3), 245-256. https://doi.org/10.1007/s00221-011-2723-y

Schütz-Bosbach, S., Avenanti, A., Aglioti, S. M., \& Haggard, P. (2009). Don’t do it! Cortical inhibition and self-attribution during action observation. Journal of Cognitive Neuroscience, 21(6), 1215-1227. https://doi.org/10.1162/jocn.2009.21068

Schütz-Bosbach, S., Mancini, B., Aglioti, S. M., \& Haggard, P. (2006). Self and other in the human motor system. Current Biology : CB, 16(18), 1830-1834. https://doi.org/10.1016/j.cub.2006.07.048

Sedda, A., Tonin, D., Salvato, G., Gandola, M., \& Bottini, G. (2016). Left caloric vestibular stimulation as a tool to reveal implicit and explicit parameters of body representation. Consciousness and Cognition, 41, 1-9. https://doi.org/10.1016/j.concog.2016.01.012

Seinfeld, S., Arroyo-Palacios, J., Iruretagoyena, G., Hortensius, R., Zapata, L. E., Borland, D., de Gelder, B., Slater, M., \& Sanchez-Vives, M. V. (2018). Offenders become the victim in virtual reality: impact of changing perspective in domestic violence. Scientific Reports, 8(1), 2692. https://doi.org/10.1038/s41598-018-19987-7

Serino, A., Alsmith, A., Costantini, M., Mandrigin, A., Tajadura-Jimenez, A., \& Lopez, C. (2013). Bodily ownership and self-location: Components of bodily self-consciousness. Consciousness and Cognition, 22(4), 1239-1252. https://doi.org/10.1016/j.concog.2013.08.013

Serino, S., Pedroli, E., Keizer, A., Triberti, S., Dakanalis, A., Pallavicini, F., Chirico, A., \& Riva, G. (2016). Virtual Reality Body Swapping: A Tool for Modifying the Allocentric Memory of the Body. Cyberpsychology, Behavior, and Social Networking, 19(2), 127- 
133. https://doi.org/10.1089/cyber.2015.0229

Serino, S., Polli, N., \& Riva, G. (2019). From avatars to body swapping: The use of virtual reality for assessing and treating body-size distortion in individuals with anorexia. Journal of Clinical Psychology, 75(2), 313-322. https://doi.org/10.1002/jclp.22724

Serino, S., Scarpina, F., Dakanalis, A., Keizer, A., Pedroli, E., Castelnuovo, G., Chirico, A., Catallo, V., Di Lernia, D., \& Riva, G. (2018). The Role of Age on Multisensory Bodily Experience: An Experimental Study with a Virtual Reality Full-Body Illusion.

Cyberpsychology, Behavior, and Social Networking, 21(5), 304-310. https://doi.org/10.1089/cyber.2017.0674

Sforza, A., Bufalari, I., Haggard, P., \& Aglioti, S. M. (2010). My face in yours: Visuo-tactile facial stimulation influences sense of identity. Social Neuroscience, 5(2), 148-162. https://doi.org/10.1080/17470910903205503

Slater, M., Perez-Marcos, D., Ehrsson, H. H., \& Sanchez-Vives, M. V. (2009). Inducing illusory ownership of a virtual body. Frontiers in Neuroscience, 3(2), 214-220. https://doi.org/10.3389/neuro.01.029.2009

Slater, M., \& Sanchez-Vives, M. V. (2016). Enhancing Our Lives with Immersive Virtual Reality. Frontiers in Robotics and AI, 3, 74. https://doi.org/10.3389/FROBT.2016.00074

Smit, M., Kooistra, D. I., van der Ham, I. J. M., \& Dijkerman, H. C. (2017). Laterality and body ownership: Effect of handedness on experience of the rubber hand illusion. Laterality, 22(6), 703-724. https://doi.org/10.1080/1357650X.2016.1273940

Solcà, M., Ronchi, R., Bello-Ruiz, J., Schmidlin, T., Herbelin, B., Luthi, F., Konzelmann, M., Beaulieu, J. Y., Delaquaize, F., Schnider, A., Guggisberg, A. G., Serino, A., \& Blanke, O. (2018). Heartbeat-enhanced immersive virtual reality to treat complex regional pain syndrome. Neurology, 91(5), e1-e11. https://doi.org/10.1212/WNL.0000000000005905

Spychala, N., Debener, S., Bongartz, E., Müller, H. H. O., Thorne, J. D., Philipsen, A., \& Braun, N. (2020). Exploring Self-Paced Embodiable Neurofeedback for Post-stroke Motor Rehabilitation. Frontiers in Human Neuroscience, 13, 17. https://doi.org/10.3389/fnhum.2019.00461

Tagini, S., Scarpina, F., Bruni, F., Scacchi, M., Mauro, A., \& Zampini, M. (2020). The virtual hand illusion in obesity: Dissociation between multisensory interactions supporting illusory experience and self-location recalibration. Multisensory Research, 33(3), 337361. https://doi.org/10.1163/22134808-20191425

Tajadura-Jiménez, A., Banakou, D., Bianchi-Berthouze, N., \& Slater, M. (2017). Embodiment 
in a Child-Like Talking Virtual Body Influences Object Size Perception, SelfIdentification, and Subsequent Real Speaking. Scientific Reports, 7(1), 1-12. https://doi.org/10.1038/s41598-017-09497-3

Taylor, S. E., \& Brown, J. D. (1988). Illusion and Well-Being: A Social Psychological Perspective on Mental Health. Psychological Bulletin, 103(2), 193-210. https://doi.org/10.1037/0033-2909.103.2.193

Thakkar, K.N., Nichols, H. S., Gilling McIntosh, L., Cochran, C. J., \& Park, S. (2011).

Disturbed sense of body ownership in schizophrenia. Schizophrenia Bulletin, 37, 230-231. https://doi.org/10.1093/schbul/sbq173 LK - http://sfx39bic.hosted.exlibrisgroup.com/sfxbic3/?sid=EMBASE\&sid=EMBASE\&issn=05867614 \&id=doi:10.1093\%2Fschbul\%2Fsbq173\&atitle=Disturbed + sense + of + body + ownership + in + schizophrenia\&stitle=Schizophr.+Bull.\&title=Schizophrenia+Bulletin\&volume=37\&issu $\mathrm{e}=\&$ spage $=230 \&$ epage $=231 \&$ aulast $=$ Thakkar \&aufirst $=$ Katharine + Natasha \&auinit $=$ K.N.\& aufull $=$ Thakkar $+K \cdot N . \& \operatorname{coden}=\&$ isbn $=\&$ pages $=230$ -

$231 \&$ date $=2011 \&$ auinit $1=$ K\&auinitm $=\mathrm{N}$

Thakkar, Katharine N., Nichols, H. S., McIntosh, L. G., \& Park, S. (2011). Disturbances in body ownership in schizophrenia: Evidence from the rubber hand illusion and case study of a spontaneous out-of-body experience. PLoS ONE, 6(10).

https://doi.org/10.1371/journal.pone.0027089

Themelis, K., \& Newport, R. (2018). An investigation of contextual factors in the application of multisensory illusions for analgesia in hand osteoarthritis. Rheumatology Advances in Practice, 2(2), 1-10. https://doi.org/10.1093/rap/rky019

Thieme, H., Bayn, M., Wurg, M., Zange, C., Pohl, M., \& Behrens, J. (2013). Mirror therapy for patients with severe arm paresis after stroke - A randomized controlled trial. Clinical Rehabilitation, 27(4), 314-324. https://doi.org/10.1177/0269215512455651

Thøgersen, M., Hansen, J., Arendt-Nielsen, L., Flor, H., \& Petrini, L. (2018). Removing ownlimb visual input using mixed reality (MR) produces a "telescoping" illusion in healthy individuals. Behavioural Brain Research, 347, 263-271. https://doi.org/10.1016/j.bbr.2018.03.024

Tidoni, E., Fusco, G., Leonardis, D., Frisoli, A., Bergamasco, M., \& Aglioti, S. M. (2014). Illusory movements induced by tendon vibration in right- and left-handed people. Experimental Brain Research, 233(2), 375-383. https://doi.org/10.1007/s00221-014-41218 
Tieri, G., Gioia, A., Scandola, M., Pavone, E. F., \& Aglioti, S. M. (2017). Visual appearance of a virtual upper limb modulates the temperature of the real hand: a thermal imaging study in Immersive Virtual Reality. European Journal of Neuroscience, 45(9), 1141-1151. https://doi.org/10.1111/ejn.13545

Tilak, M., Isaac, S. A., Fletcher, J., Vasanthan, L. T., Subbaiah, R. S., Babu, A., Bhide, R., \& Tharion, G. (2016). Mirror Therapy and Transcutaneous Electrical Nerve Stimulation for Management of Phantom Limb Pain in Amputees - A Single Blinded Randomized Controlled Trial. Physiotherapy Research International, 21(2), 109-115. https://doi.org/10.1002/pri.1626

Tsakiris, M. (2010). My body in the brain: a neurocognitive model of body-ownership. Neuropsychologia, 48(3), 703-712. https://doi.org/10.1016/j.neuropsychologia.2009.09.034

Tsakiris, M., Carpenter, L., James, D., \& Fotopoulou, A. (2010). Hands only illusion: multisensory integration elicits sense of ownership for body parts but not for noncorporeal objects. Experimental Brain Research. Experimentelle Hirnforschung. Expérimentation Cérébrale, 204(3), 343-352. https://doi.org/10.1007/s00221-009-2039-3

Tsay, A., Allen, T. J., Proske, U., \& Giummarra, M. J. (2015). Sensing the body in chronic pain: A review of psychophysical studies implicating altered body representation. Neuroscience and Biobehavioral Reviews, 52, 221-232. https://doi.org/10.1016/j.neubiorev.2015.03.004

Tung, M. L., Murphy, I. C., Griffin, S. C., Alphonso, A. L., Hussey-Anderson, L., Hughes, K. E., Weeks, S. R., Merritt, V., Yetto, J. M., Pasquina, P. F., \& Tsao, J. W. (2014). Observation of limb movements reduces phantom limb pain in bilateral amputees. Annals of Clinical and Translational Neurology, 1(9), 633-638. https://doi.org/10.1002/acn3.89

Vallar, G., \& Ronchi, R. (2009). Somatoparaphrenia: a body delusion. A review of the neuropsychological literature. Experimental Brain Research. Experimentelle Hirnforschung. Expérimentation Cérébrale, 192(3), 533-551. https://doi.org/10.1007/s00221-008-1562-y

Valzolgher, C., Mazzurega, M., Zampini, M., \& Pavani, F. (2018). Incongruent multisensory stimuli alter bodily self-consciousness: Evidence from a first-person perspective experience. Acta Psychologica, 191, 261-270. https://doi.org/10.1016/j.actpsy.2018.09.009

van der Hoort, B., Guterstam, A., \& Ehrsson, H. H. (2011). Being Barbie: the size of one's own body determines the perceived size of the world. PloS One, 6(5), e20195. 
https://doi.org/10.1371/journal.pone.0020195

Van Doorn, G., De Foe, A., Wood, A., Wagstaff, D., \& Hohwy, J. (2018). Down the rabbit hole: assessing the influence of schizotypy on the experience of the Barbie Doll Illusion. Cognitive Neuropsychiatry, 23(5), 284-298. https://doi.org/10.1080/13546805.2018.1495623

Van Haren, N., Prikken, M., van der Weiden, A., Baalbergen, H., Hillegers, M., Aarts, H., \& Kahn, R. (2018). O2.5. Multisensory Integration Underlying Body Ownership in Schizophrenia and Individuals At Familial Risk To Develop Psychosis: a Study Using the Rubber Hand Illusion Paradigm. Schizophrenia Bulletin, 44(suppl_1), S77-S77. https://doi.org/10.1093/schbul/sby015.195

Weber, L. M., Nilsen, D. M., Gillen, G., Yoon, J., \& Stein, J. (2019). Immersive Virtual Reality Mirror Therapy for Upper Limb Recovery after Stroke: A Pilot Study. American Journal of Physical Medicine and Rehabilitation, 98(9), 783-788. https://doi.org/10.1097/PHM.0000000000001190

Westerhof, G. J., \& Keyes, C. L. M. (2010). Mental illness and mental health: The two continua model across the lifespan. Journal of Adult Development, 17(2), 110-119. https://doi.org/10.1007/s10804-009-9082-y

Wu, C. Y., Huang, P. C., Chen, Y. T., Lin, K. C., \& Yang, H. W. (2013). Effects of mirror therapy on motor and sensory recovery in chronic stroke: A randomized controlled trial. Archives of Physical Medicine and Rehabilitation, 94(6), 1023-1030. https://doi.org/10.1016/j.apmr.2013.02.007

Yildirim, M., \& Kanan, N. (2016). The effect of mirror therapy on the management of phantom limb pain. Agri, 28(3), 127-134. https://doi.org/10.5505/agri.2016.48343

Zeller, D., \& Hullin, M. (2018). Spatial attention and the malleability of bodily self in the elderly. Consciousness and Cognition, 59, 32-39. https://doi.org/10.1016/j.concog.2018.01.006

Zeller, D., Litvak, V., Friston, K. J., \& Classen, J. (2014). Sensory Processing and the Rubber Hand Illusion- An Evoked Potential Study. Journal of Cognitive Neuroscience. https://doi.org/10.1162/jocn

Zopf, R., Contini, E., Fowler, C., Mondraty, N., \& Williams, M. A. (2016). Body distortions in Anorexia Nervosa: Evidence for changed processing of multisensory bodily signals. Psychiatry Research, 245, 473-481. https://doi.org/10.1016/j.psychres.2016.09.003

Zopf, R., Truong, S., Finkbeiner, M., Friedman, J., \& Williams, M. A. (2011). Viewing and 
feeling touch modulates hand position for reaching. Neuropsychologia, 49(5), 1287-1293. https://doi.org/10.1016/j.neuropsychologia.2011.02.012 